ARTICLE

\title{
Chromosomally unstable tumor cells specifically require KIF18A for proliferation
}

Carolyn Marquis ${ }^{1}$, Cindy L. Fonseca', Katelyn A. Queen ${ }^{1}$, Lisa Wood', Sarah E. Vandal ${ }^{1}$, Heidi L. H. Malaby ${ }^{1}$, Joseph E. Clayton ${ }^{2} \&$ Jason Stumpff (i) ${ }^{1 凶}$

Chromosomal instability (CIN) is a hallmark of tumor cells caused by changes in the dynamics and control of microtubules that compromise the mitotic spindle. Thus, CIN cells may respond differently than diploid cells to treatments that target mitotic spindle regulation. Here, we test this idea by inhibiting a subset of kinesin motor proteins involved in mitotic spindle control. KIF18A is required for proliferation of CIN cells derived from triple negative breast cancer or colorectal cancer tumors but is not required in near-diploid cells. Following KIF18A inhibition, CIN tumor cells exhibit mitotic delays, multipolar spindles, and increased cell death. Sensitivity to KIF18A knockdown is strongly correlated with centrosome fragmentation, which requires dynamic microtubules but does not depend on bipolar spindle formation or mitotic arrest. Our results indicate the altered spindle microtubule dynamics characteristic of CIN tumor cells can be exploited to reduce the proliferative capacity of CIN cells.

\footnotetext{
${ }^{1}$ Department of Molecular Physiology and Biophysics, University of Vermont, Burlington, VT, USA. ${ }^{2}$ BioTek Instruments Inc, Winooski, VT, USA.

凶email: jstumpff@uvm.edu
} 
G enetic instability is a common feature of tumor cells, and a large number of tumor cells exhibit frequent loss or gain of chromosomes ${ }^{1}$. This chromosomal instability (CIN) is primarily attributable to defects leading to abnormal interactions between chromosomes and mitotic spindle microtubules, which in turn increase chromosome segregation errors ${ }^{2-7}$. While CIN contributes to tumor progression, heterogeneity, drug resistance, and metastasis, it has been proposed that the same properties driving instability could provide an Achilles' heel for CIN cellspecific targeted therapies ${ }^{1,8,9}$. Compared to chromosomally stable cells, CIN cells display increased spindle microtubule polymerization and reduced turnover of the attachments between spindle microtubules and kinetochores, which are specialized protein structures that assemble at the centromeric regions of mitotic chromosomes ${ }^{2,3}$. Thus, CIN cells may be particularly vulnerable to anti-mitotic therapies that target the microtubule cytoskeleton.

Consistent with this idea, microtubule-targeting agents are effective therapeutics for a wide variety of tumors ${ }^{10}$. Paclitaxel, a microtubule-stabilizing drug routinely utilized to treat solid tumors, was originally demonstrated to induce cytotoxicity by preventing cells from completing mitosis ${ }^{11}$. However, due to adverse side effects associated with the broad inhibition of microtubule function, significant effort has been made to identify mitotic regulators that could be targeted with lower toxicity in cancer patients. While drugs targeting proteins essential for mitosis have shown promise in preclinical models, they have been largely unsuccessful in clinical trials ${ }^{12}$. One explanation for the apparent paradox presented by failed mitotic targeting strategies and the effective therapeutic results seen with paclitaxel is that paclitaxel may not kill tumor cells in vivo simply by preventing mitotic progression. This idea is supported by work demonstrating that clinically relevant doses of paclitaxel induce abnormal, multipolar divisions in tumors, rather than preventing mitotic division altogether ${ }^{11,13}$. Furthermore, paclitaxel leads to the induction of micronuclei due to chromosome segregation errors, which may activate innate immune pathways ${ }^{14}$. Thus, efforts to mimic the effects of paclitaxel on mitotic cells need to be refocused toward identifying proteins that can be targeted to disrupt normal bipolar divisions, ideally in a tumor cell-specific manner. Ideal targets would reduce tumor cell proliferation by inducing mitotic defects in cancer cells with minimal toxic effects in normal cells.

Here, we test the hypothesis that altered mitotic microtubule dynamics in CIN cells may confer sensitivity to the inhibition of proteins that regulate microtubule dynamics or generate forces within mitotic spindles. We focus our efforts on kinesin motors known to regulate spindle microtubule dynamics and mechanics that are also largely dispensable for division in diploid somatic cells. Our results demonstrate that the majority of CIN tumor cells tested depend on the kinesin-8 motor KIF18A to maintain bipolar spindle integrity and promote proliferation.

\section{Results}

KIF18A is required for the proliferation of CIN tumor cells. To compare the impacts of altered kinesin function in cells with or without CIN, we measured cell proliferation in both stable, diploid breast epithelial MCF10A cells and the chromosomally unstable triple-negative breast cancer (TNBC) cell lines MDAMB-231, MDA-MB-468, and HCC1806 ${ }^{15}$ following knockdown (KD) of kinesin motor proteins. Specifically, the effects of KIF18A, KIF18B, KIF4A, KIF22/KID, and KIF2C/MCAK KD were determined (Supplementary Fig. 1). Cell proliferation was measured using an automated, high-contrast bright-field microscopy-based kinetic assay (Supplementary Fig. 2). KIF18A $\mathrm{KD}$ significantly reduced the proliferation of all three TNBC cell lines but did not affect the growth of diploid MCF10A cells (Fig. 1a, b). To determine if this trend holds in other tumor cell types, we measured proliferation in colorectal cancer (CRC) cells categorized as displaying either chromosomal instability (CIN) or microsatellite instability (MSI), a form of genomic instability arising from defective DNA repair in near-diploid tumor cells ${ }^{16}$. KIF18A KD significantly reduced the proliferation of two CIN cell lines but had minor effects on the proliferation of MSI cells (Fig. 1c and Supplementary Fig. 1). The proliferation of chromosomally unstable HeLa Kyoto cells, which have been used extensively for studies of KIF18A function, was also reduced by KIF18A KD (Fig. 1c). CIN cells sensitive to KIF18A knockdown exhibited increased cell death following KIF18A siRNA treatment, while near-diploid HCT116 and MCF10A cells did not (Fig. 2). Taken together, the diploid cells tested here did not require KIF18A to proliferate, while the majority of CIN tumor cells displayed a dependence on KIF18A for efficient growth and survival.

Loss of KIF18A induces a prolonged mitotic delay in CIN tumor cells. KIF18A is required for chromosome alignment in all cells but also promotes spindle assembly checkpoint satisfaction and progression through mitosis in some cell types ${ }^{17-23}$. To determine if proliferation defects seen in KIF18A-depleted CIN cells correlate with KIF18A's role in promoting timely metaphase-to-anaphase transitions, we compared the effects of KIF18A KD on mitotic progression in CIN cells and near-diploid cells. KIF18A KD led to an increase in the percentage of mitotic CIN cells but did not significantly alter the percentage of mitotic cells within MCF10A or non-CIN CRC cell populations (Fig. 3a-c and Supplementary Fig. 3). Quantification of mitotic duration revealed that all cell types displayed a significant increase in the amount of time required to progress from nuclear envelope breakdown (NEB) to anaphase onset (AO) following KIF18A KD (Fig. 3d-f). Consistent with previous work, the magnitude and variance of mitotic delays were larger in KIF18A KD CIN tumor cells than diploid (MCF10A) or near-diploid cells (HCT116) (Fig. 3d) ${ }^{19-21,24,25}$. In addition, the cell types most sensitive to KIF18A KD contained a significant subpopulation of cells that failed to complete mitosis during the imaging studies and were arrested for up to $20 \mathrm{~h}$ (Fig. 2e). SW480 CIN cells, which were not dependent on KIF18A for proliferation, did not display extended mitotic arrest, suggesting that they are able to compensate for the loss of KIF18A in order to complete cell division. These data suggest that proliferation defects in KIF18Adependent CIN cells may stem from defects that prevent subpopulations of cells from completing mitosis.

KIF18A-dependent CIN cells form multipolar spindles. Analyses of mitotic spindles in KIF18A KD cells revealed that KIF18A-dependent CIN lines display a significant increase in multipolar spindles compared to non-KIF18A-dependent cell lines (Fig. 4a, b). Multipolar spindles were defined as spindles containing more than two microtubule-organizing centers with foci of the pericentriolar component $\gamma$-tubulin. Interestingly, the fold-increase in multipolar spindles following KIF18A KD was inversely proportional to the fold decrease in proliferation for each cell type (Fig. 4c). These data indicate that mitotic spindle assembly is abnormal in most KIF18A-dependent CIN cells. However, HeLa cells, which displayed reduced proliferation following KIF18A KD, displayed a slightly different spindle phenotype. A fraction of HeLa cells exhibited fragmented $\gamma$-tubulin in response to KIF18A KD, but overall did not display a significant increase in multipolar spindles (Fig. $4 \mathrm{~b}$ and Supplementary Fig. 4a). These observations suggest that although 
a
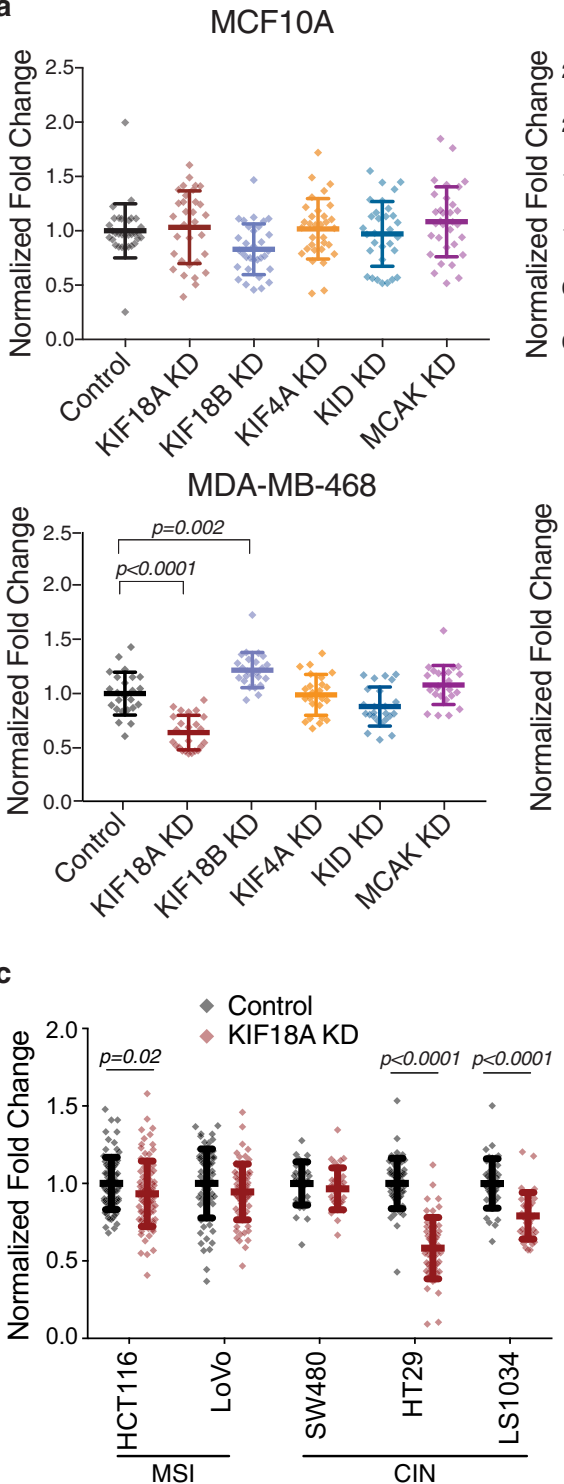

MDA-MB-231

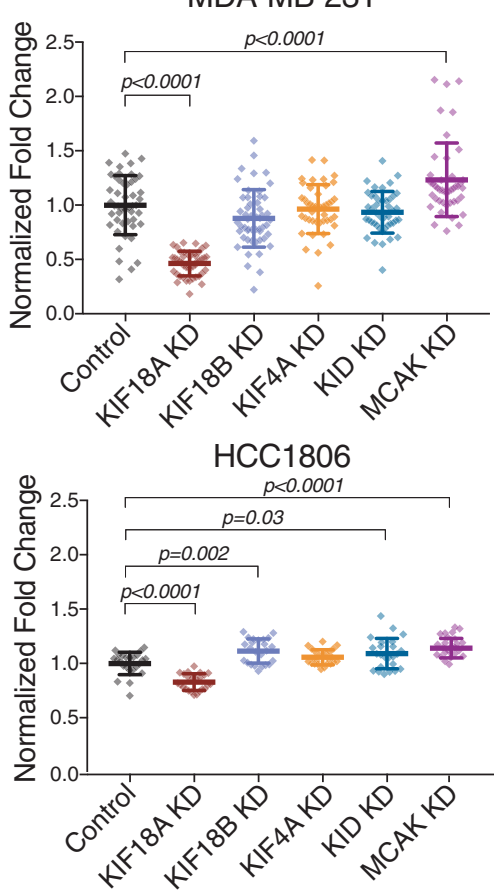

d

HeLa Kyoto

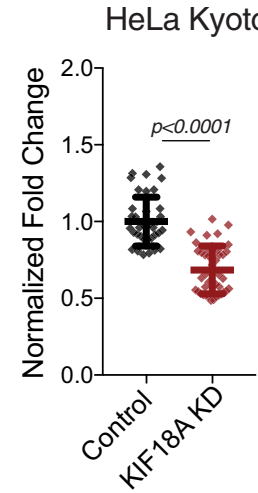

b
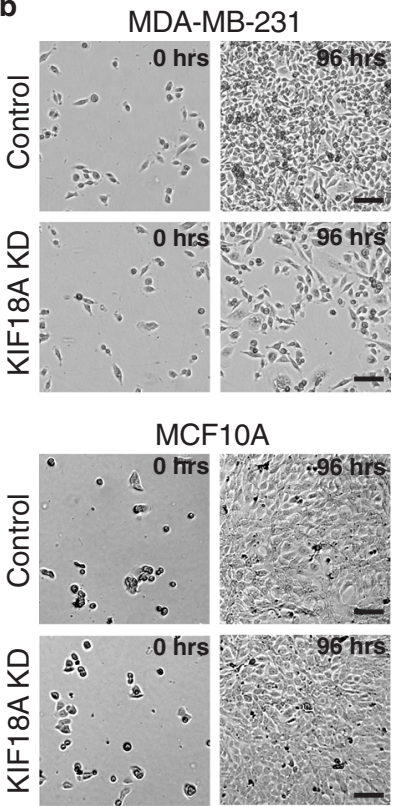

Fig. 1 KIF18A is required for the proliferation of chromosomally unstable cells. a Fold change in cell density (cells $/ \mathrm{mm}^{2}$ ) after $96 \mathrm{~h}$ in the indicated cell lines following knockdown (KD) of kinesin proteins. Data are normalized to cells treated with control siRNA. Raw cell counts for proliferation assays are available in Source Data. $n$ (wells of cells/independent experiments) =24/3 (MCF10A, MDA-MB-468, and HCC1806), 64/5 (MDA-MB-231 control and KIF18A KD), 36/3 (MDA-MB-231 KIF18B, KIF4A, KIF22, and MCAK KD). b Representative images of MDA-MB-231 (from five experiments) and MCF10A cells (from three experiments) treated with either control or KIF18A siRNA. Scale bars are 100 microns. c Normalized fold change in cell density (cells/ $\mathrm{mm}^{2}$ ) of $\mathrm{MSI}$ and $\mathrm{CIN}$ colorectal cancer cell lines after $96 \mathrm{~h}$ or until cells in the control populations reached confluence. $n$ (wells of cells/independent experiments) $=88 / 6$ (HCT116); 104/5 (LoVo control KD) and 128/5 (LoVo KIF18A KD); 92/5 (SW480); 44/3 (HT29 control KD) and 48/3 (HT29 KIF18A KD); and 32/3 (LS1034). d Normalized fold change in cell density (cells $/ \mathrm{mm}^{2}$ ) of HeLa Kyoto cells after $96 \mathrm{~h}$ or until cells in the control populations reached confluence. $n=40$ wells of cells from two independent experiments. Data were analyzed using a one-way ANOVA with post hoc Tukey's test to compare the control to each of the knockdown conditions (a) or with an unpaired, two-tailed $t$ test (c, d). All graphs show mean \pm SD and individual data points. $P$ values $<0.05$ from the indicated statistical tests are displayed on plots.

abnormal $\gamma$-tubulin distribution occurs in KIF18A KD HeLa cells, other mechanisms may be keeping the spindles from becoming multipolar. To investigate this further, we examined the effects of KIF18A KD in combination with previously validated siRNAs targeting either CLASP1 or HSET, which have roles in maintaining centrosome integrity and clustering supernumerary centrosomes ${ }^{26-30}$. HeLa cells treated with siRNAs against both KIF18A and either HSET or CLASP1 exhibited a significant increase in multipolar spindles with more than two $\gamma$-tubulin foci compared to cells treated with siRNAs against a single target (Supplementary Fig. 4b). These co-depletion phenotypes were rescued when GFP-KIF18A was inducibly expressed at levels similar to endogenous KIF18A, which were also sufficient to rescue mitotic progression defects caused by KIF18A KD (Supplementary Fig. 4b-e). Notably, the combined effects of KIF18A and CLASP1 on spindle morphology were not specific for HeLa cells. Similar results were seen in SW480 cells, suggesting this cell type also relies on other mechanisms for centrosome integrity in the absence of KIF18A (Supplementary Fig. 4f). Together, these data suggest a function for KIF18A in preserving centrosome integrity that is essential for the maintenance of spindle bipolarity in the majority of CIN cells tested.

Loss of KIF18A function could lead to multipolar spindles by promoting centrosome amplification, cytokinesis failure, centriole 


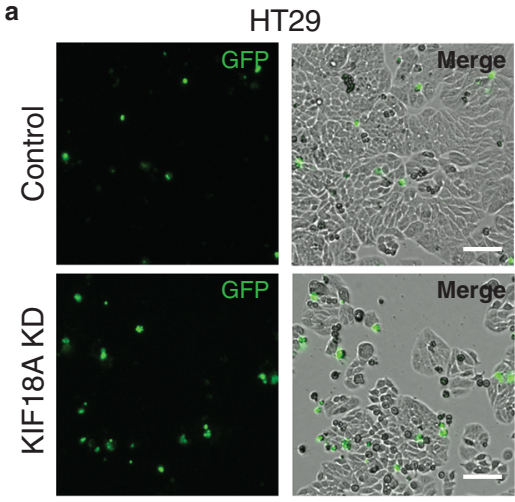

b

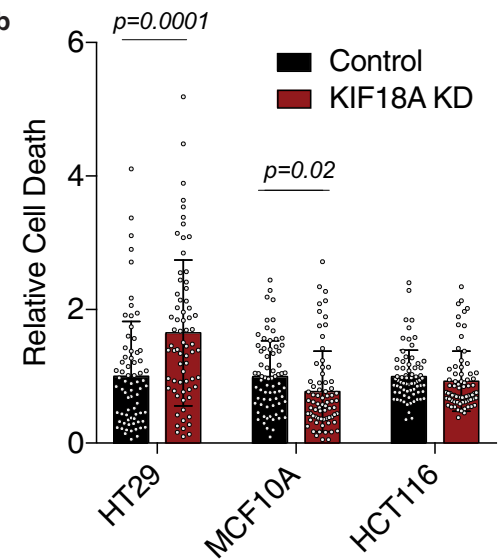

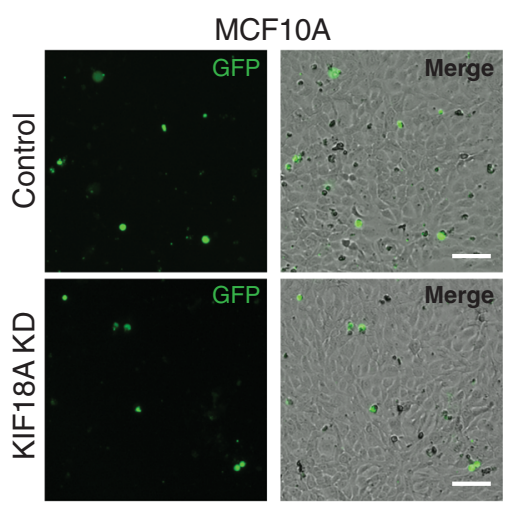

c

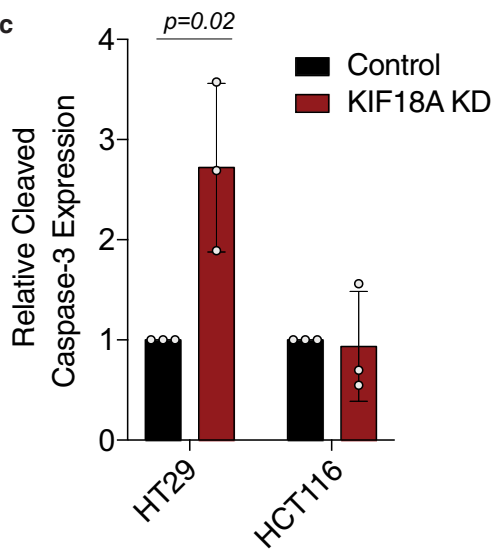

d

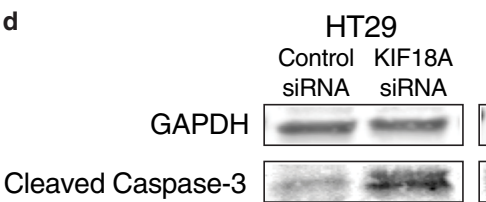

Cleaved Caspase-3
HCT116

Control KIF18A

SiRNA SIRNA

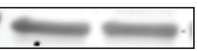

$37 \mathrm{kD}$

$17 \& 19 \mathrm{kD}$

Fig. 2 KIF18A depletion increases cell death in CIN cells. a Representative images (from three independent experiments) of HT29 and MCF10A cells labeled with Celltox Green cytotoxicity dye (green) 5 days after siRNA transfection. Scale bars are 100 microns. b Relative cell death calculated as the normalized ratio of the change in Celltox-stained cell density to the change in total cell density over $96 \mathrm{~h} . n$ (wells of cells/independent experiments) $=72 /$ 3 for MCF10A and 71/3 for HT29 and HCT116. c Relative expression of cleaved-caspase 3 measured via western blot for each condition. Results are from three independent experiments. d Western blot showing representative cleaved-caspase-3 (CC3) expression levels. All graphs show mean \pm SD and individual data points. Data were analyzed by unpaired, two-tailed $t$ test. $P$ values $<0.05$ are displayed.

disengagement, or pericentriolar material (PCM) fragmentation $^{31}$. To distinguish among these mechanisms, we analyzed the number and organization of centrioles within multipolar spindles in MDA-MB-231 cells (Fig. 4d-f). The majority of spindles $(\sim 75 \%)$ in both control and KIF18A KD cells contained four centrioles, indicating that centrosome amplification and cytokinesis failure do not significantly contribute to spindle defects in KIF18A KD cells.

The average distance between paired centrioles was increased in multipolar KIF18A KD cells compared to those in bipolar spindles but was comparable to the average distance measured in multipolar spindles treated with control siRNA (Fig. 4f). However, $\sim 60 \%$ of multipolar KIF18A KD cells exhibited $\gamma$-tubulincontaining microtubule-organizing centers without centrioles (Fig. 4e). A similar trend was observed in HT29 cells following KIF18A KD (Supplementary Fig. 5). Furthermore, live imaging of KIF18A-depleted MDA-MB-231 cells labeled with siR-tubulin revealed an increase in spindle pole fragmentation events but not the number of cells entering mitosis with multiple poles compared to control siRNA-treated cells (Fig. 4g-i and Supplementary
Movies 1-3). These data suggest that KIF18A KD primarily leads to multipolar spindles by inducing PCM fragmentation.

KIF18A KD induces multipolar spindles in CIN cells independently of mitotic delay. The fragmentation of centrosomes and formation of multipolar spindles following KIF18A KD could result from abnormal spindle forces caused by altered microtubule dynamics or as a secondary effect of an extended mitotic delay $^{31}$. To determine if a mitotic delay is required for multipolar spindle formation following KIF18A KD, we analyzed spindle morphology in MDA-MB-231 cells depleted of both KIF18A and MAD2, which is required for spindle assembly checkpointdependent mitotic arrest ${ }^{32}$. KIF18A/MAD2 KD cells displayed a reduced mitotic index but a similar level of multipolar spindles compared to KIF18A KD cells (Fig. 5a, b). Spindle pole splitting in live cells occurred at a range of times after mitotic entry in KIF18A KD cells and at times shortly after NEB in KIF18A/ MAD2 KD cells (Fig. 5c-e and Supplementary Movie 4). The significant decrease in multipolar KIF18A/MAD2 KD cells 
a
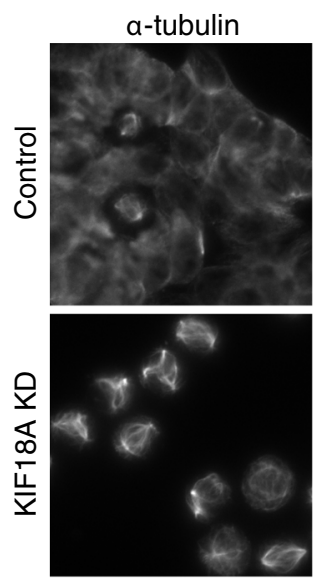

c

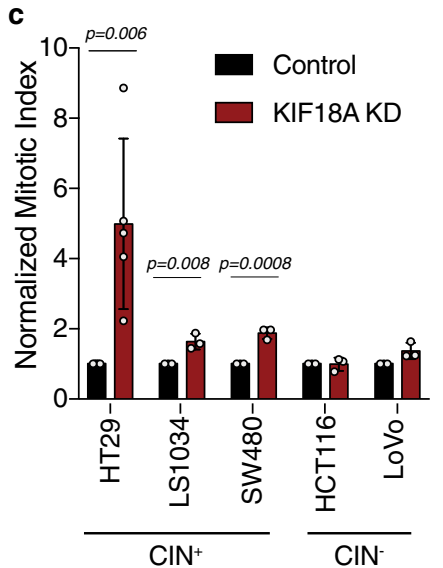

f
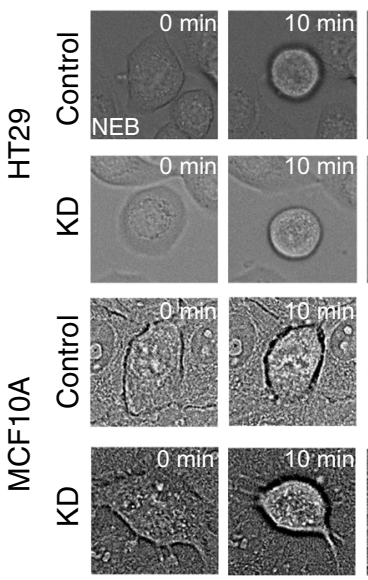

HT29 $\left(\mathrm{CIN}^{+}\right)$
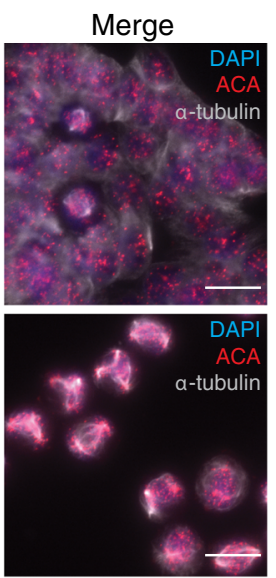

b
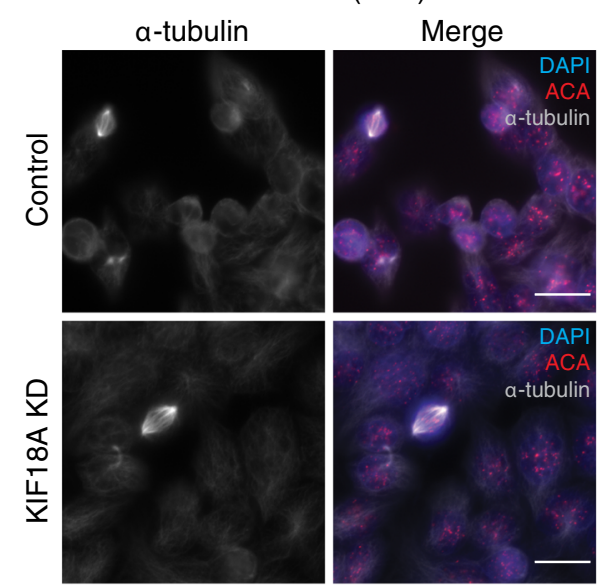

d
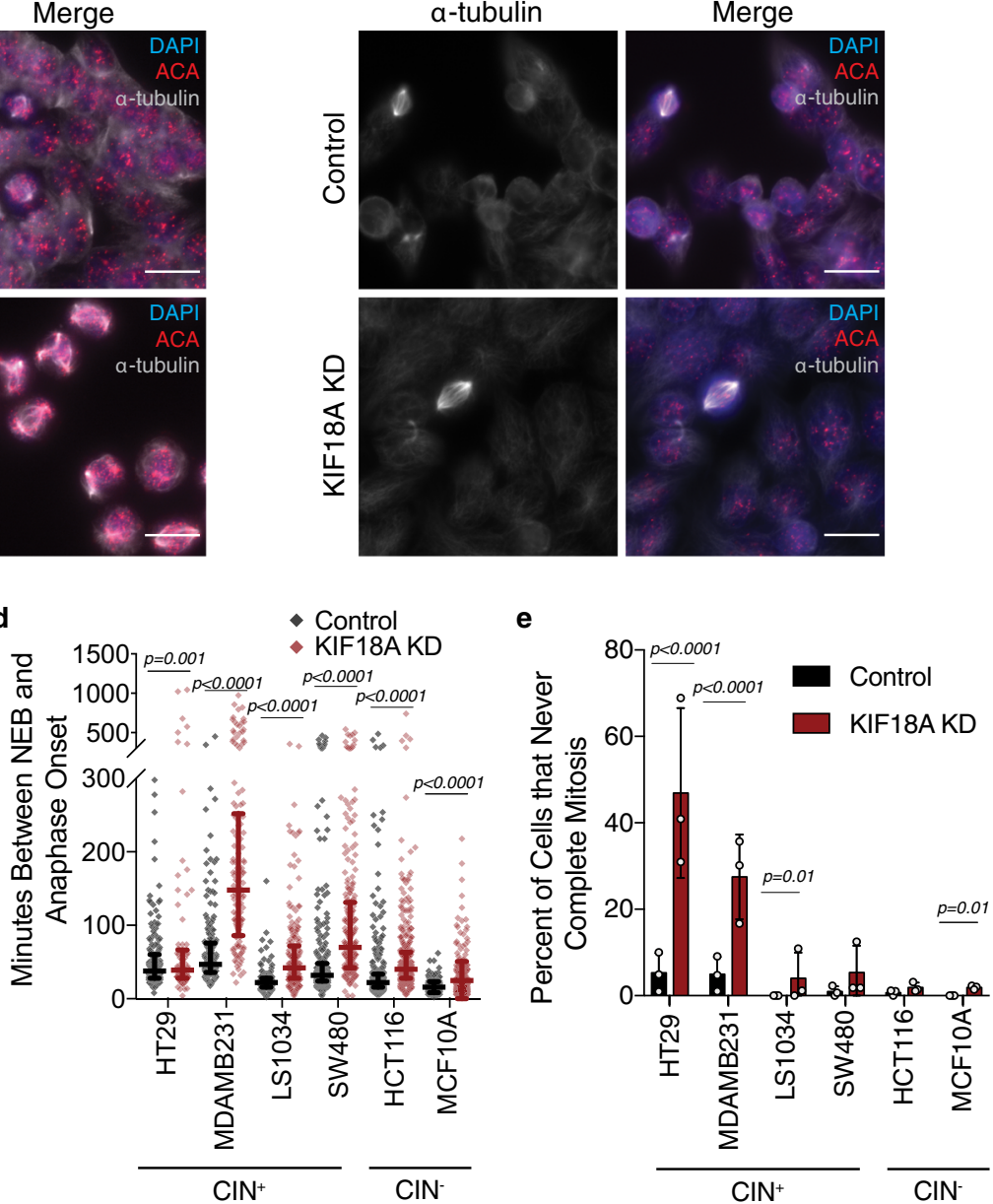

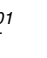

(1)
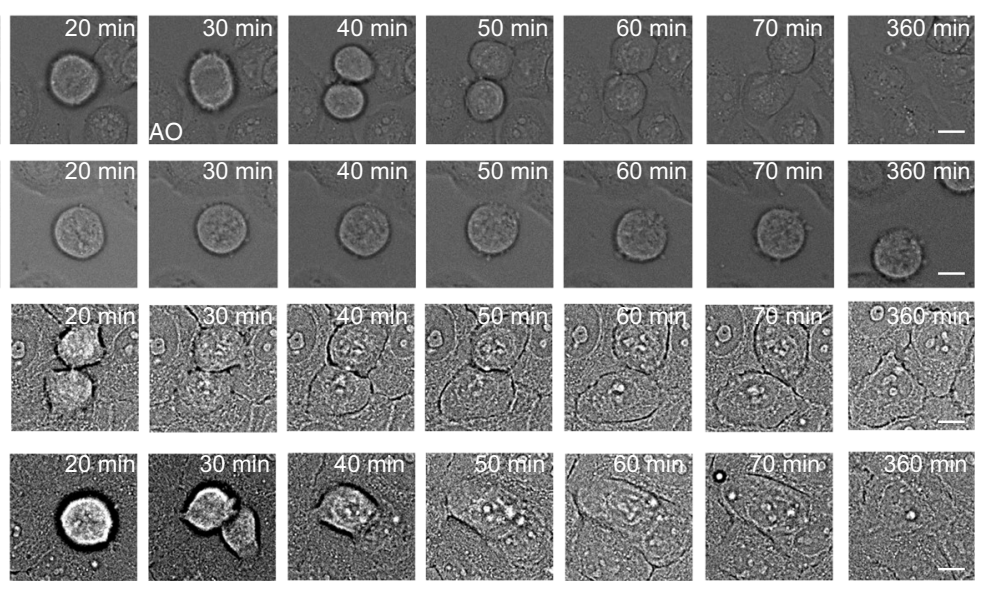

Fig. 3 KIF18A depletion causes mitotic arrest in CIN cancer cells. a, b Representative images from five and three independent experiments of $\mathrm{HT} 29$ (a) or HCT116 cells, respectively, (b) treated with control or KIF18A siRNAs. DNA (DAPI, blue), microtubules ( $\alpha$-tubulin, white), and centromeres (ACA, red) are labeled. Scale bars are 10 microns. c Percentage of mitotic cells (mitotic index) observed in fixed populations of control or KIF18A siRNA-treated CRC cells. $n=$ three independent biological samples per condition for LS1034, SW480, HCT116, and LoVo; $n=5$ for HT29. Data were analyzed via unpaired, twotailed $t$ test. d Time between nuclear envelope breakdown (NEB) and anaphase onset (AO) in control or KIF18A siRNA-treated cells. Data from three independent experiments are shown for each cell line. $n$ (control KD/KIF18A KD cells)=185/ 83 (HT29), 182/135 (MDA-MB-231), 203/196 (LS1034), 303/236 (SW480), 332/344 (HCT116), and 338/353 (MCF10A). Data were analyzed via unpaired, two-tailed $t$ test. e Percentage of control or KIF18A siRNA-treated cells that entered mitosis at least $200 \mathrm{~min}$ before the end of the movie but did not divide. Data are from the same three experiments as $\mathbf{d}$ and were analyzed by a two-sided Chi-square test. $n$ (control KD/KIF18A KD cells) =191/153 (HT29), 189/195 (MDA-MB-231), 203/202 (LS1034), 310/ 249 (SW480), 335/351 (HCT116), and 338/367 (MCF1OA). f Frames from DIC live-cell imaging of HT29 and MCF10A cells treated with control or KIF18A (KD) siRNAs, showing the progression from NEB to AO. Scale bars are 5 microns, representative of three independent experiments. All graphs show mean \pm SD and individual data points. $P$ values $<0.05$ are displayed. 

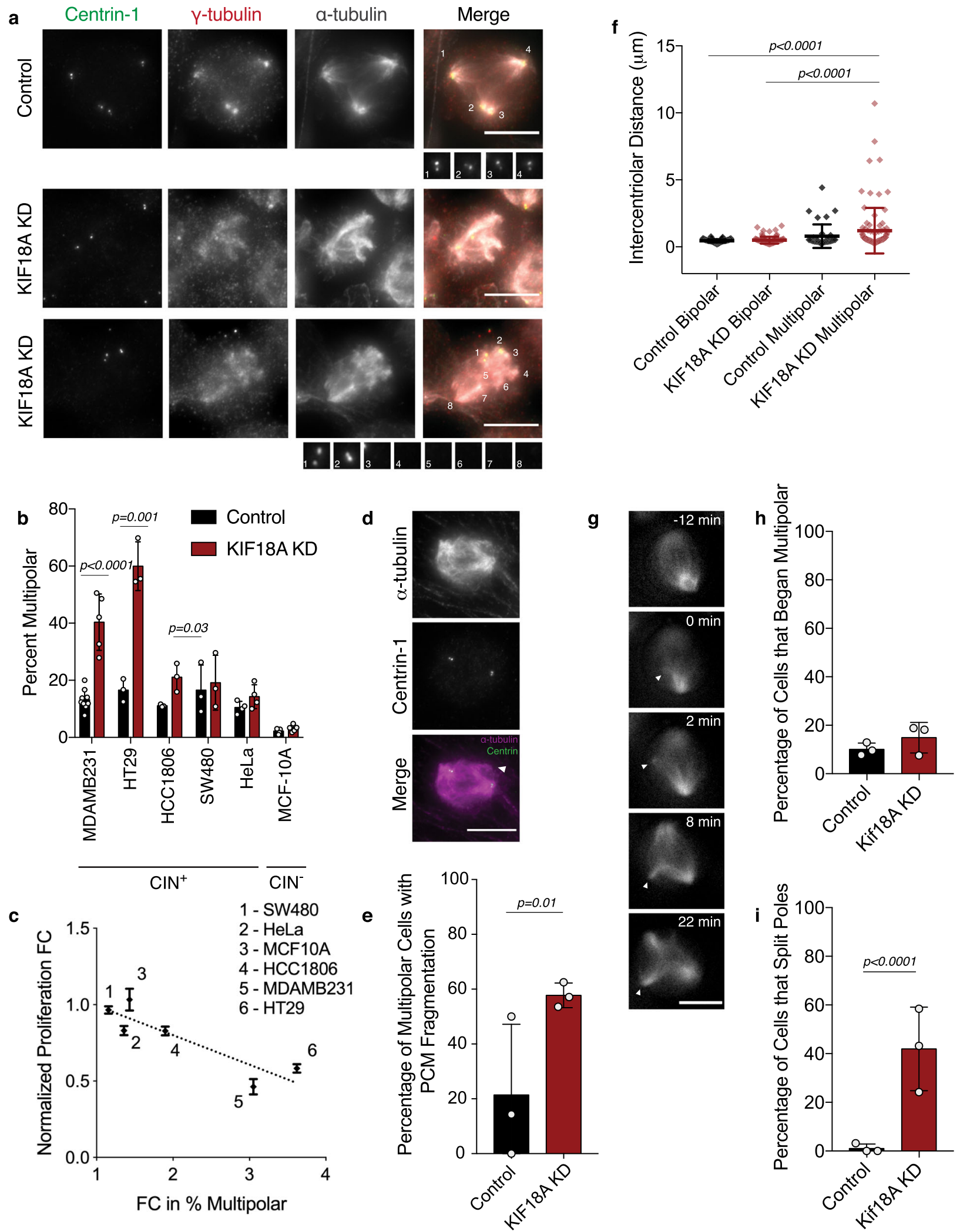

compared to KIF18A KD alone observed during live imaging may be explained by the limitations inherent to the identification of multipolar spindles in live assays, as poles must split sufficiently far apart to be completely separated in this case. Therefore, the live approach is likely to underestimate the actual time to splitting and percentage of multipolar spindles, especially in cells that exit mitosis quickly. Taken together, these data suggest that loss of
KIF18A leads to spindle pole fragmentation in CIN cells and that this defect does not require, but may be enhanced by, a mitotic delay.

Centrosome fragmentation in KIF18A $\mathrm{KD}$ cells requires dynamic microtubules. KIF18A functions to suppress microtubule 
Fig. 4 Loss of KIF18A causes centrosome fragmentation in MDA-MB-231 cells. a Representative images from five independent experiments of MDAMB-231 cells treated with either control (top) or KIF18A (bottom) siRNAs. Pericentriolar material ( $\gamma$-tubulin, red) is numbered to show poles with and without centrioles (centrin-1, green). Microtubules ( $\alpha$-tubulin) are labeled in white. b Percent of mitotic cells from each indicated cell line with multipolar spindles following treatment with either control or KIF18A siRNA. $n=9$ (control KD) and 5 (KIF18A KD) independent experimental replicates for MDAMB-231. $n=3$ (HT29, HCC1806, SW480), 4 (HeLa), and 5 (MCF10A) independent experimental replicates. Data were analyzed via unpaired, two-tailed $t$ test. c Plot of multipolar spindle percentage as a function of fold change (FC) in cell number for the indicated cell lines following KIF18A KD. R-squared value is 0.79 using a linear regression model. $\mathbf{d}$ Representative images from three independent experiments of an MDA-MB-231 cell with a third pole ( $\gamma$ tubulin, magenta) lacking centrin-1 (green). e Percent of multipolar MDA-MB-231 cells in mitosis with fragmented pericentriolar material (PCM), as indicated by the presence of $\gamma$-tubulin puncta lacking centrin-1 puncta. $n=18$ (control KD) and 58 (KIF18A KD) multipolar cells from three independent experiments. Data were analyzed via a two-sided Chi-square test. $\mathbf{f}$ Intercentriolar distance measurements (in microns) for MDA-MB-231 cells in each indicated category. $n=81$ (control KD bipolar), 38 (control KD multipolar), 94 (KIF18A KD bipolar), and 91 (KIF18A KD multipolar) centriole pairs from three independent experiments. Data were analyzed via one-way ANOVA with post hoc Tukey's test for multiple comparisons. $\mathbf{g}$ Representative still frames from three independent experiments of a live MDA-MB-231 KIF18A KD cell labeled with siR-tubulin. Arrows indicate pole splitting and separation. h, i Percent of live, siR-tubulin-labeled MDA-MB-231 cells that (h) enter mitosis with more than two spindle poles or (i) split and separate spindle poles during mitosis. $n=126$ (control KD) and 172 (KIF18A KD) cells from three independent experiments. Data were analyzed by a two-sided Chi-square test. All graphs show mean \pm SD and individual data points. $P$ values $<0.05$ are displayed. All scale bars are 10 microns.

a

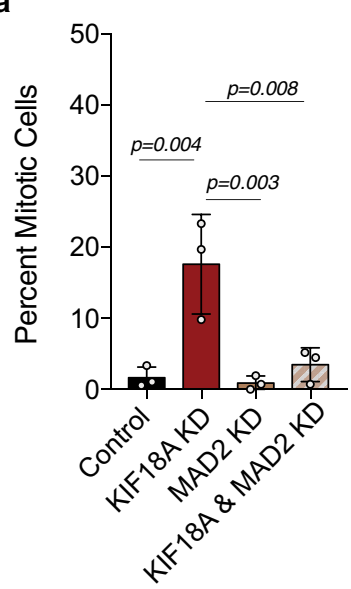

C

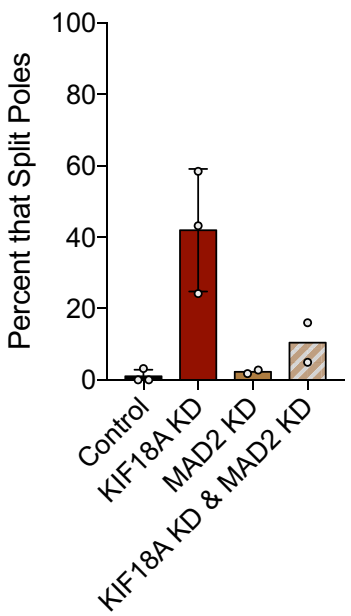

b

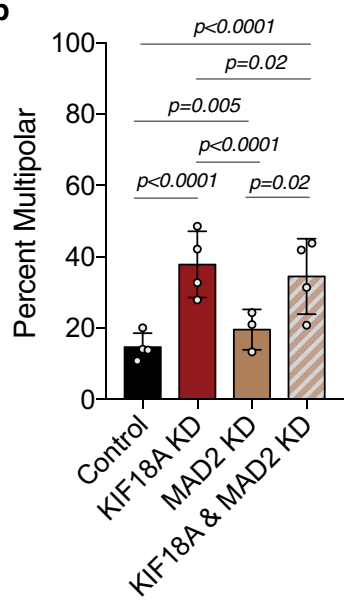

d

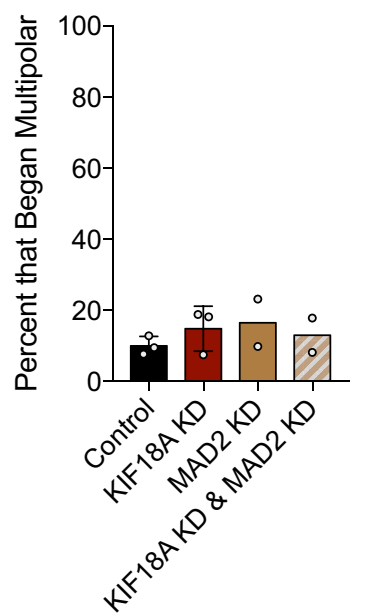

e

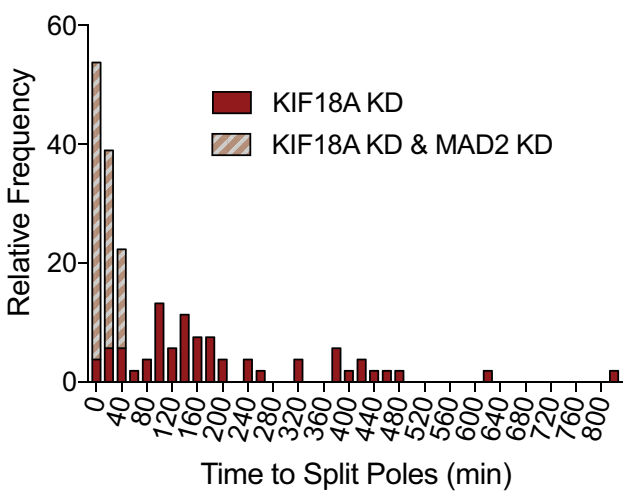

$\mathbf{f}$

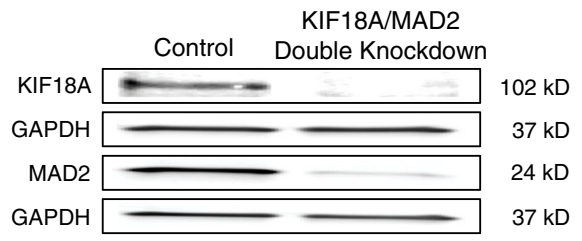

Fig. 5 Spindle checkpoint inhibition rescues mitotic arrest but not multipolar spindle formation caused by KIF18A KD. a, b Percent of fixed MDA-MB231 cells (a) in mitosis or (b) with multipolar spindles after the indicated siRNA knockdown (KD). $n=3$ independent biological samples for all conditions in a, and $n=3$ (MAD2 KD) or 4 (control, KIF18A, and KIF18A \& MAD2 KD) in b. Data were analyzed via one-way ANOVA with post hoc Tukey's test for multiple comparisons (a) or two-sided Chi-square test (b). $P$ values $<0.05$ are displayed. c, $\mathbf{d}$ Percent of live, siR-tubulin-labeled MDA-MD-231 cells that (c) split poles during mitosis or (d) entered mitosis with more than two spindle poles. $n$ (number of cells/number of independent experiments) $=126 / 3$ (control), 172/3 (KIF18A), 127/2 (MAD2), and 117/2 (KIF18A and MAD2). All bars represent mean, and error bars represent SD. Individual data points are shown. e Stacked histogram showing relative frequencies of the duration of time between NEB and pole splitting for siR-tubulin-labeled MDA-MB-231 cells following KIF18A KD or KIF18A and MAD2 KD. f Western blots of MAD2, KIF18A, and GAPDH following treatment with either a double dose of control siRNA or a combination of KIF18A and MAD2 siRNA. 

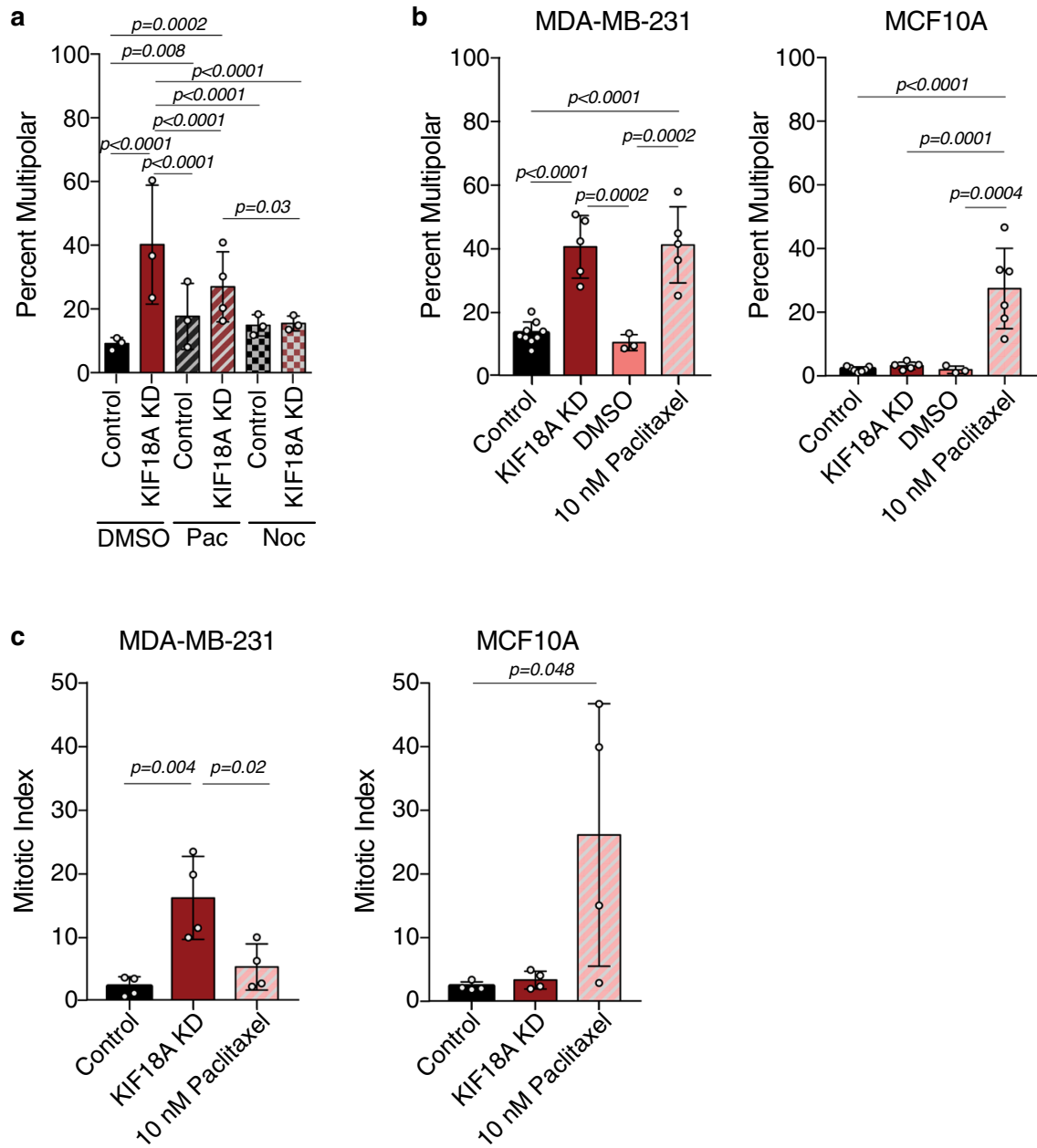

Fig. 6 KIF18A KD-induced centrosome fragmentation is reduced by paclitaxel and nocodazole. a Percent of MDA-MB-231 cells with multipolar spindles in control or KIF18A KD cells treated with either DMSO, $20 \mathrm{nM}$ Paclitaxel (Pac), or $5 \mu$ M Nocodazole (Noc) for $3 \mathrm{~h}$. $n=151$ (control KD + DMSO), 263 , (KIF18A KD + DMSO), 189 (control KD + pac), 218 (KIF18A KD + pac), 155 (control KD + noc), and 158 (KIF18A KD + noc) cells from three independent experiments. Data were analyzed via a two-sided Chi-square test. b Percentage of mitotic cells with multipolar spindles in fixed MDA-MB-231 or MCF10A after the indicated treatments. $n=9$ (control KD), 5 (KIF18A KD), 3 (DMSO), and 5 (paclitaxel) independent experimental replicates for MDA-MB-231 cells. $n=7$ (control KD), 5 (KIF18A KD), 3 (DMSO), and 6 (paclitaxel) independent experimental replicates for MCF10A cells. Data were analyzed via a two-sided Chi-square test. c Percentage of fixed MDA-MB-231 and MCF10A cells in mitosis following the indicated treatments. $n=4$ independent biological samples for all conditions. Data were analyzed via one-way ANOVA with post hoc Tukey's test for multiple comparisons. All graphs show mean \pm SD with individual data points indicated. $P$ values $<0.05$ from the indicated statistical tests are displayed.

growth within mitotic spindles ${ }^{18,33}$, suggesting that abnormal microtubule dynamics in KIF18A KD cells may contribute to centrosome fragmentation. We tested this idea by reducing microtubule polymerization or depolymerizing microtubules completely via the treatment of KIF18A KD MDA-MB-231 cells with $20 \mathrm{nM}$ paclitaxel or $5 \mu \mathrm{M}$ nocodazole, respectively ${ }^{34,35}$. KIF18A KD cells treated with either paclitaxel or nocodazole for 3 $\mathrm{h}$ before fixation displayed significantly fewer multipolar spindles than KIF18A KD cells treated with DMSO (Fig. 6a). These data indicate that dynamic microtubules are required for KIF18A KDinduced centrosome fragmentation.

Paclitaxel treatment causes an increase in multipolar spindles in both somatic diploid cells and aneuploid cancer cells ${ }^{13,36,37}$, whereas KIF18A KD preferentially affected spindle morphology in the CIN cells tested here (Fig. 4a, b). To directly compare the two treatments, we analyzed MDA-MB-231 and MCF10A cells after KIF18A KD or incubation with a clinically relevant dose of paclitaxel $(10 \mathrm{nM})$ for $24 \mathrm{~h}$ in parallel ${ }^{13}$. Paclitaxel treatment led to increased multipolar spindles and mitotic index in both MDAMB-231 cells and MCF10A cells, while KIF18A KD only induced these mitotic defects in MDA-MB-231 cells (Fig. 6b, c). Thus, paclitaxel and KIF18A KD induce a similar spindle phenotype but appear to act via antagonistic mechanisms, consistent with their opposite effects on spindle microtubule dynamics. Furthermore, sensitivity to KIF18A loss of function is higher in the CIN cell lines tested here than in near-diploid MCF10A cells.

Centrosome fragmentation in KIF18A KD cells does not require bipolar spindle formation. Our live imaging studies indicated that KIF18A KD MDA-MB-231 and HT29 cells form bipolar spindles before centrosome fragmentation occurs (Fig. 4, Supplementary Fig. 5, and Supplementary Movies 1-3). Therefore, altered microtubule dynamics in KIF18A KD cells could lead to centrosome fragmentation by disrupting the balance of pushing and pulling forces within bipolar spindles. To test this idea, we assayed the number of $\gamma$-tubulin foci in MDA-MB-231 cells treated with the KIF11 inhibitor monastrol, which induces monopolar spindles by preventing KIF11-dependent antiparallel microtubule sliding forces ${ }^{38}$. We found that centrosome 
a

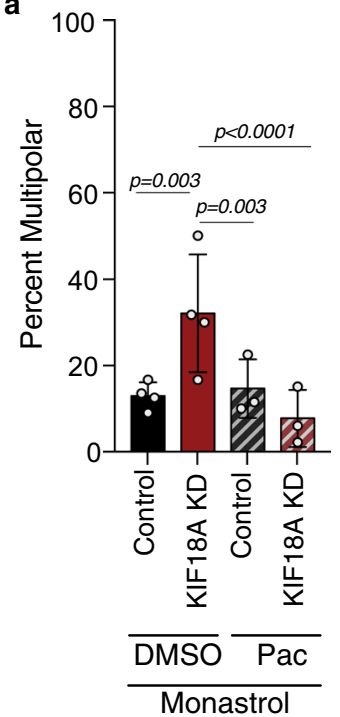

b
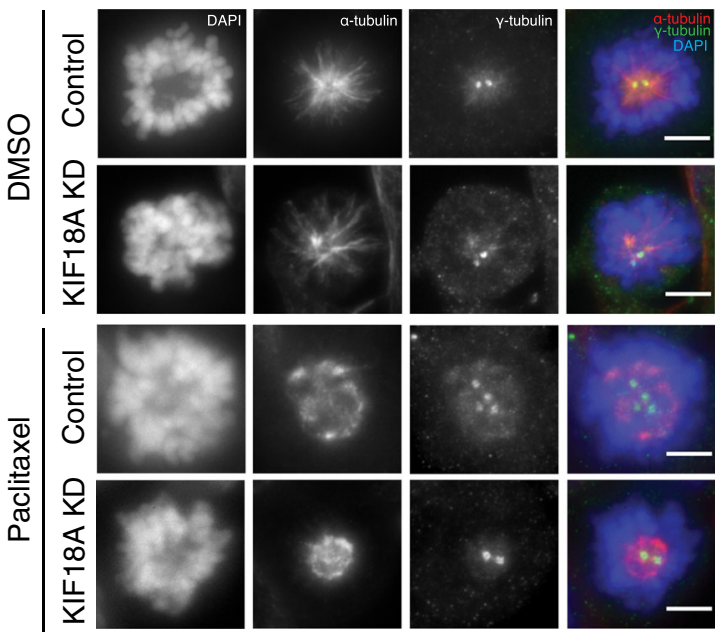

Fig. 7 Centrosome fragmentation in KIF18A KD cells does not require bipolar spindle formation. a Percent of monopolar MDA-MB-231 cells with three or more $\gamma$-tubulin puncta in control or KIF18A KD cells treated with both monastrol (20 $\mu$ M) and either DMSO or 20 nM Paclitaxel (Pac). $n$ (number of monopolar mitotic cells/number of independent experiments) $=112 / 4$ (control KD + DMSO), 102/4 (KIF18A KD + DMSO), 132/3 (control KD + Pac), and $149 / 3$ (KIF18A KD + Pac). The plot shows mean \pm SD with individual data points indicated. Data were analyzed via a two-sided Chi-square test, and $P$ values $<0.05$ are displayed. $\mathbf{b}$ Representative images (from three independent experiments) of MDA-MB-231 cells treated with $20 \mu M$ monastrol and either DMSO or $20 \mathrm{nM}$ Paclitaxel. DNA (DAPI, blue), microtubules ( $\alpha$-tubulin, red), and centrosomes ( $\gamma$-tubulin, green) are labeled. Scale bar is 5 microns.

fragmentation still occurred in monopolar KIF18A KD cells and could be reduced by co-treatment with paclitaxel (Fig. 7a, b). Live imaging of monastrol treated cells expressing RFP-pericentrin to label centrosomes revealed that centrosomes begin intact in monopolar KIF18A KD cells and subsequently fragment (Supplementary Movie 5). These data suggest that neither bipolar spindles nor the forces generated via KIF11-dependent microtubule sliding are required for centrosome fragmentation in the absence of KIF18A.

The effects of KIF18A KD are enhanced by activation of MCAK/KIF2C. In addition to suppressing the growth of kinetochore microtubules, KIF18A is required to decrease kinetochore-microtubule turnover ${ }^{18,39}$. Thus, we reasoned that increased kinetochore-microtubule turnover may contribute to the prolonged mitotic delays and destabilized spindles observed in KIF18A KD CIN cells. To test this, we treated cells with a small molecule (UMK57) that promotes kinetochore-microtubule turnover by increasing the activity of the depolymerizing kinesin MCAK/KIF2C ${ }^{40}$. Treatment of KIF18A-depleted MDA-MB231 cells with UMK57 $(500 \mathrm{nM})$ decreased proliferation and significantly increased the mitotic index compared to KIF18A KD cells treated with DMSO (Fig. $8 \mathrm{a}-\mathrm{c}$ ). The same concentration of UMK57 had no impact on the proliferation of control siRNAtreated cells (Fig. 8b). UMK57 treatment of KIF18A KD cells also led to a small but significant increase in multipolar spindles, and this effect was replicated in cells with increased global MCAK/ KIF2C activity, due to overexpression of mCherry-MCAK, or increased MCAK/KIF2C activity at centromeres, due to expression of mCherry-CPB-MCAK ${ }^{41}$ (Fig. $8 \mathrm{~d}$ and Supplementary Fig. 6a). Furthermore, in live cells labeled with siR-tubulin, codepletion of both KIF18A and KIF2C reduced multipolar spindle formation compared to depletion of KIF18A alone, while KIF18A KD cells treated with UMK57 displayed increased spindle pole fragmentation (Fig. 8e, f, Supplementary Fig. 6b, c, and Supplementary Movie 6). These data indicate that loss of KIF18A function combined with increased KIF2C function, particularly at centromeres, synergistically disrupts mitotic progression and spindle bipolarity.

\section{Discussion}

Our data support a model in which the altered microtubule dynamics in mitotic CIN cells make them particularly dependent on KIF18A to reduce kinetochore-microtubule turnover and limit microtubule growth. In the absence of KIF18A activity, centrosome fragmentation occurs and mitotic progression is slowed or prevented. Importantly, these effects were not observed in the near-diploid, chromosomally stable cells tested here, which is consistent with previous observations that reduction of KIF18A activity leads to longer spindle assembly checkpoint-dependent delays in cancer cells than diploid somatic cells ${ }^{17,19-23}$. KIF18A is also largely dispensable for the proliferation of diploid somatic cells in vivo but is necessary for tumor growth. Kif18a mutant mice display an early growth delay and germline development defects ${ }^{19,42}$. However, the growth of both induced CRC and xenografted TNBC tumors in mouse models are dependent on KIF18A ${ }^{43,44}$. Thus, KIF18A may be an effective target to specifically inhibit the growth of CIN tumor cells, while inducing relatively low toxicity in somatic, diploid cells.

These data raise the important question of why CIN cells would depend more on KIF18A for successful mitosis than chromosomally stable cells. CIN cells exhibit increased rates of spindle microtubule polymerization and altered turnover of kinetochore microtubules 2,3 , which may confer an enhanced dependence on KIF18A's function to suppress microtubule growth $^{18,45,46}$. Our results suggest that in the absence of KIF18A activity, maintenance of kinetochore-microtubule attachments and centrosome integrity are compromised in CIN cells, subsequently leading to extended mitotic arrest and centrosome fragmentation. These phenotypes were suppressed by treatments that reduce microtubule dynamics (nocodazole, paclitaxel, and KIF2C $\mathrm{KD})$ and were enhanced by treatments that increase microtubule dynamics (UMK57, KIF2C overexpression) or compromise centrosome integrity (CLASP1 or HSET KD). Taken together, these 
a
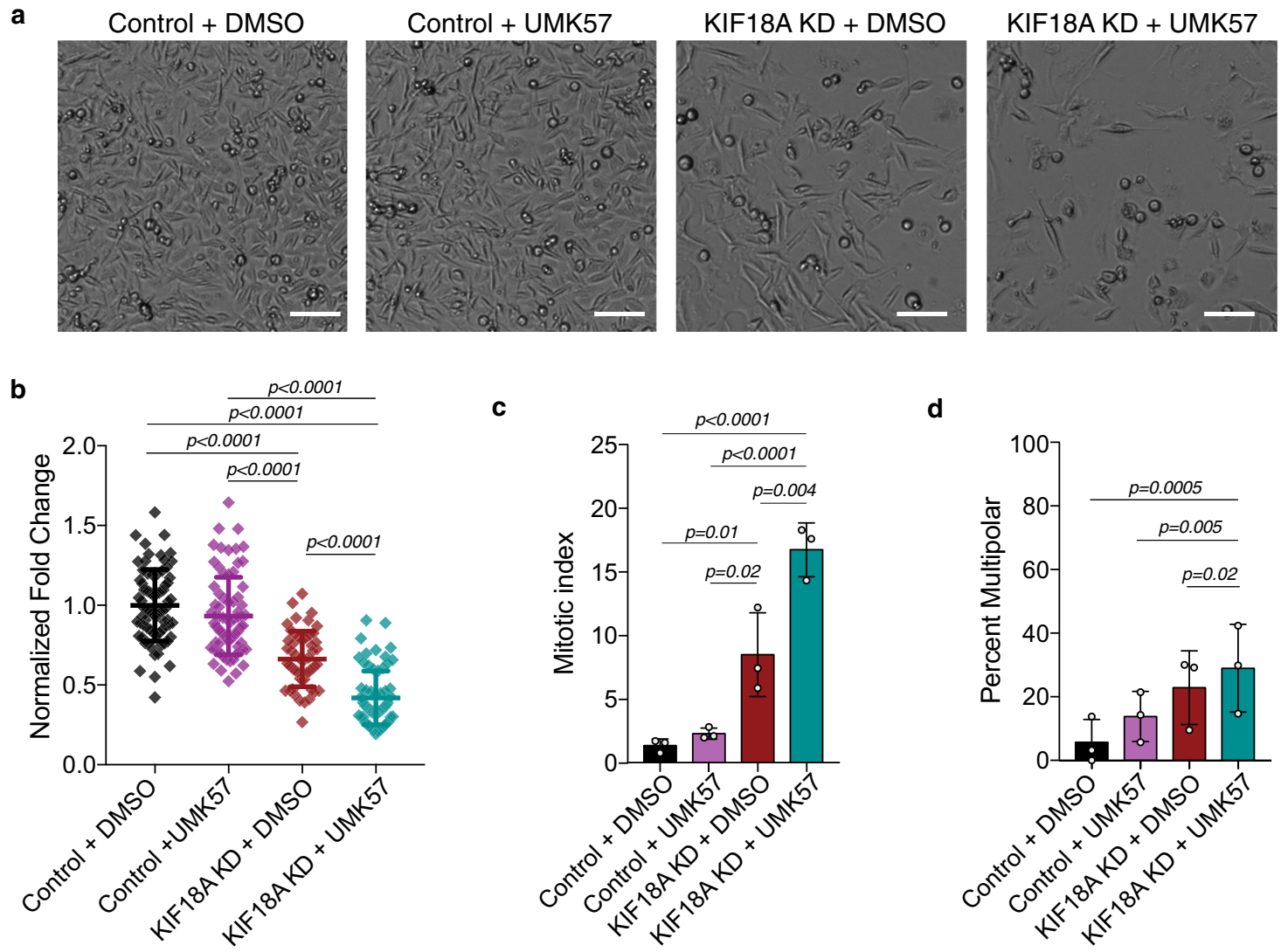

c

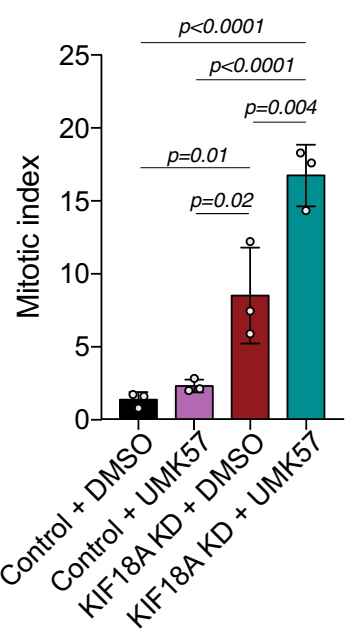

d

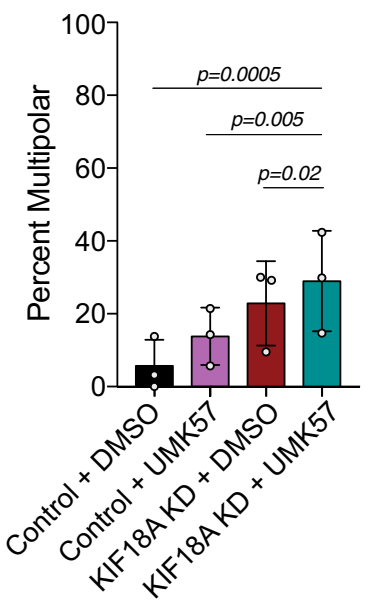

e

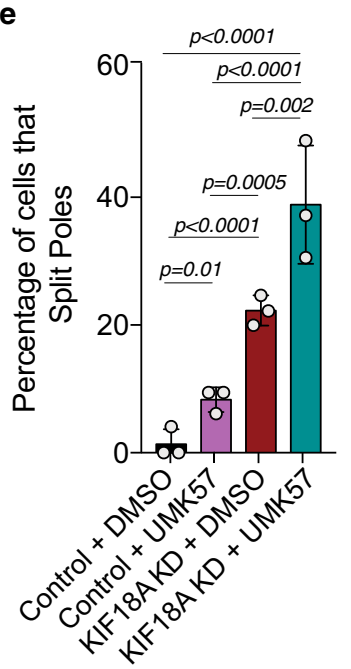

f

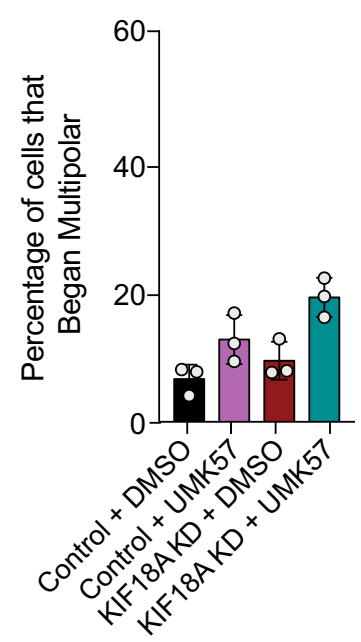

Fig. 8 KIF18A KD-induced defects depend on dynamic microtubules and are enhanced by increased KIF2C/MCAK activity. a Representative images (from three independent experiments) of MDA-MB-231 cell density $96 \mathrm{~h}$ after the start of high-contrast bright-field imaging. Cells were treated with either control or KIF18A siRNAs in combination with DMSO or $500 \mathrm{nM}$ UMK57. Scale bar is 100 microns. b Fold change in cell density after $96 \mathrm{~h}$ in MDA-MB231 cells treated with the specified siRNAs and either $500 \mathrm{nM}$ UMK57 or DMSO. $n=76$ (control KD + DMSO), 52 (control KD + UMK57), 76 (KIF18A KD + DMSO), and 68 (KIF18A KD + UMK57) wells of cells from three independent experiments. Data were analyzed via one-way ANOVA with post hoc Tukey's test for multiple comparisons. c, d Percent of total mitotic cells (c) and mitotic cells with multipolar spindles (d) in fixed populations after the indicated treatments. $n=3$ independent experimental replicates per condition. Data were analyzed via one-way ANOVA with post hoc Tukey's test for multiple comparisons. e, $\mathbf{f}$ Percent of live, siR-tubulin-labeled MDA-MB-231 cells that (e) split poles or (f) entered mitosis with more than two spindle poles after the indicated treatments. $n=126$ (control KD + DMSO), 158 (control KD + UMK57), 172 (KIF18A KD + DMSO), and 152 (KIF18A KD + UMK57) mitotic cells from three independent experiments. Data were analyzed via a two-sided Chi-square test. All graphs show mean \pm SD and individual data points. $P$ values $<0.05$ are displayed. 


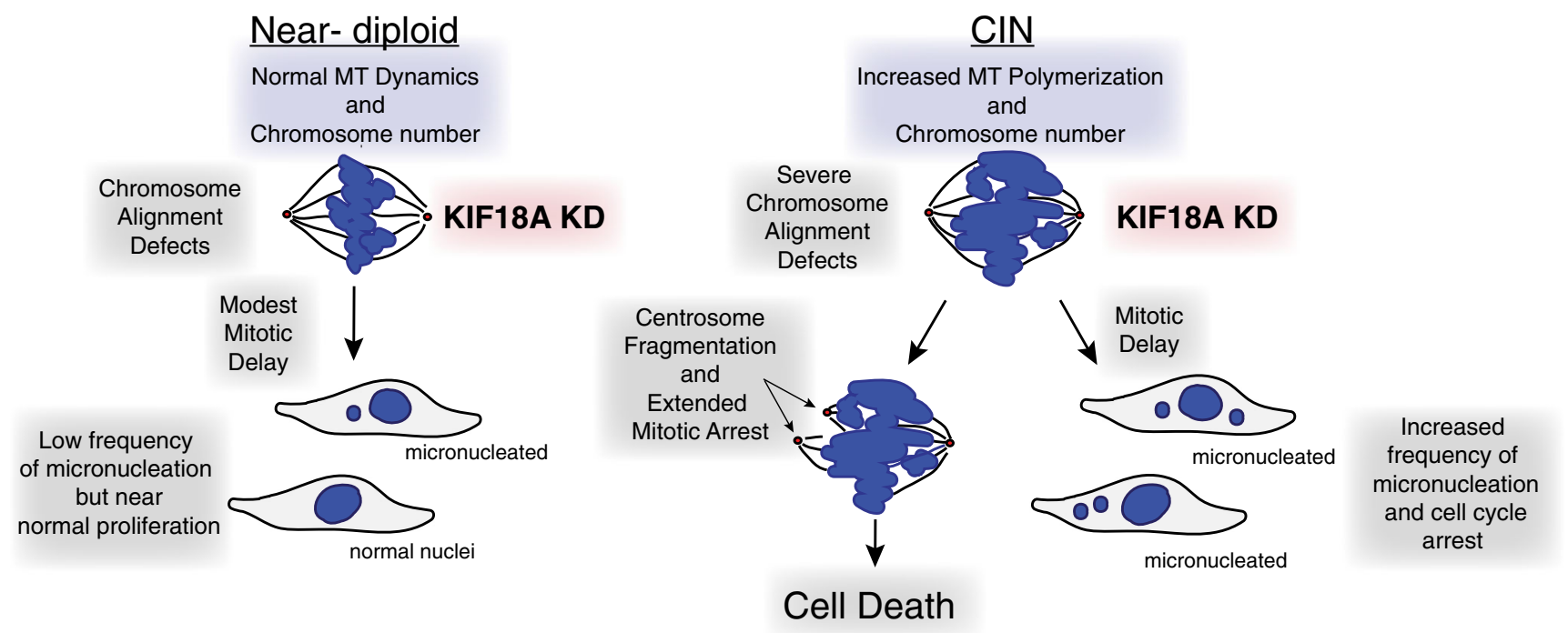

Fig. 9 Model for selective dependence of CIN cells on KIF18A function. Schematic summarizing the differential responses of CIN and near-diploid cells to KIF18A knockdown (KD). Increased microtubule polymerization rates in CIN cells may sensitize them to further deregulation of microtubule dynamics caused by loss of KIF18A function, leading to force imbalances that disrupt the integrity of the spindle. See "Discussion" for additional detail.

data support a model in which the combined effects of increased microtubule polymerization in CIN cells and loss of KIF18A's microtubule growth suppression create a force imbalance within spindles that reduces centrosome integrity (Fig. 9). In addition, this force imbalance does not require a bipolar spindle configuration or KIF11-dependent microtubule sliding within the spindle, as indicated by centrosome fragmentation in monastrol treated cells.

We also observed that a significant fraction of CIN cells is able to complete mitosis after a mitotic delay. Loss of KIF18A could also slow the growth of this population by inducing chromosome segregation errors. KIF18A KD increases the frequency of micronucleus formation as a result of chromosome alignment defects, and these micronucleated cells display reduced proliferation ${ }^{20}$. In support of this idea, recent work indicates that the frequency of KIF18A KD-dependent micronuclei is increased in cells with elevated chromosome number and correlates with reduced proliferation in aneuploid cells ${ }^{47,48}$.

The mitotic defects induced by KIF18A KD, multipolar spindles and micronuclei, are similar to those proposed to underlie the anti-tumor activity of paclitaxel ${ }^{11,14}$, yet KIF18A KD and paclitaxel have antagonistic effects on microtubule dynamics and lead to reduced multipolar spindle formation when combined. This suggests that increases or decreases in microtubule dynamics may compromise spindle integrity in CIN tumor cells, an idea supported by observations that multipolar spindles form in cells with dampened microtubule dynamics due to KIF18A overexpression $^{45}$. Our tests of other kinesins that control spindle microtubule dynamics and chromosome movements suggest the observed dependence of CIN cells on KIF18A is unique. In agreement, two recent, large-scale bioinformatics studies identified Kif18A, but not other kinesins, as a gene specifically required for the growth of cells displaying aneuploidy or whole-genome duplication ${ }^{47,48}$. Thus, KIF18A represents a potential target for exploiting vulnerabilities specific to a significant fraction of tumor cells displaying CIN or aneuploidy.

\section{Methods}

Cell culture and transfections. HT29, LoVo, SW480, LS1034, HCC1806, HCT116, MCF10A, MDA-MB-231, and MDA-MB-468 cells were purchased from ATCC. HeLa Kyoto acceptor cells for recombination-mediated cassette exchange were previously described. All cell lines were validated by STR DNA fingerprinting using the Promega GenePrint 10 System according to the manufacturer's instructions (Promega \#B9510). HT29, LoVo, SW480, MDA-MB-231, and MDAMB-468 cells were cultured in DMEM/F-12 medium (Gibco) supplemented with $10 \%$ FBS (Gibco) and 1\% penicillin/streptomycin (pen/strep). LS1034 and HCC1806 cells were cultured in RPMI 1640 medium (Gibco) with 10\% FBS and $1 \%$ pen/strep. HCT116 cells were cultured in McCoys 5 A media (Gibco) with $10 \%$ FBS and $1 \%$ pen/strep and MCF10A cells were cultured in DMEM/F-12 supplemented with $5 \%$ horse serum (Gibco), $20 \mathrm{ng} / \mathrm{ml}$ epidermal growth factor, $0.5 \mu \mathrm{g} / \mathrm{ml}$ hydrocortisone, $100 \mathrm{ng} / \mathrm{ml}$ cholera toxin, $10 \mu \mathrm{g} / \mathrm{ml}$ insulin, and $1 \%$ pen/strep. To inhibit specific kinesins, cells were treated with 5 pmol siRNA with Lipofectamine RNAiMAX Transfection Reagent (Invitrogen) in Opti-MEM Reduced-Serum Media (Gibco). Specific siRNAs include pools of Silencer and Silencer Select KIF18A (Invitrogen), KIF18B (Dharmacon), KIF4A (Invitrogen), KID/KIF22 (Invitrogen), MCAK/KIFf2C (Dharmacon), MAD2 (Invitrogen), CLASP1 (Dharmacon), HSET/KIFC1 (Dharmacon), and pools of scrambled-sequence negative control siRNAs (Invitrogen). For double knockdowns involving the inhibition of two proteins, Lipofectamine RNAiMAX was used at a lowered concentration $(0.7 \times$ the concentration used for single knockdowns) to mitigate toxicity.

Generation of inducible HeLa Kyoto cell line. HeLa Kyoto cells that inducibly express GFP or GFP-KIF18A were generated via recombination-mediated cassette exchange, as previously described ${ }^{49}$. Briefly, a wild-type KIF18A siRNA and puromycin-resistant plasmid were developed containing LoxP sites for recombination-mediated cassette exchange. The LoxP containing plasmid was then transfected into HeLa Kyoto acceptor cells (a kind gift from Ryoma Ohi's lab) ${ }^{50}$, and cells that had undergone recombination were selected via puromycin. The open reading frame for a KIF18A wild-type siRNA-resistant construct ${ }^{51}$ and pEM791 vector ${ }^{49}$ were amplified with primers designed for Gibson Assembly (New England BioLabs, see Supplemental Table 1 for primer sequences). After confirming the correct sequence of the Gibson assembled plasmid, recombination was achieved by transfecting the acceptor cells with the KIF18A plasmid and recombinase using an LTX transfection (Thermo Fisher Scientific). Recombination was initially selected with $1 \mu \mathrm{g} / \mathrm{mL}$ puromycin for $48 \mathrm{~h}$ followed by a stricter selection with $2 \mu \mathrm{g} / \mathrm{mL}$ puromycin for $48 \mathrm{~h}$ prior to switching back to $1 \mu \mathrm{g} / \mathrm{mL}$ puromycin The resulting wild-type KIF18A-inducible cell line was maintained in MEM Alpha (Life Technologies) with $10 \%$ FBS (Life Technologies) and $1 \mu \mathrm{g} / \mathrm{mL}$ puromycin at $37^{\circ} \mathrm{C}, 5 \% \mathrm{CO}_{2}$.

Drug treatments. For experiments involving siRNA knockdown followed by drug treatment, the indicated concentrations of paclitaxel (Selleck Chemicals), nocodazole (Selleck Chemicals), and/or monastrol (Selleck Chemicals) were added to cells $24 \mathrm{~h}$ after siRNA treatment. Three hours after drug addition, cells were either fixed and stained for immunofluorescence imaging or imaged live in a glass-bottom 24-well dish. To compare the effects of paclitaxel treatment to the effects of KIF18A KD in MDA-MB-231 and MCF10A cell lines, $10 \mathrm{nM}$ of paclitaxel was added to cells $24 \mathrm{~h}$ before fixing and staining for immunofluorescence imaging.

Proliferation and cytotoxicity assays. Cells were imaged in either a 96- or 24well dish every 2 or $2 \mathrm{~h}$ for $96 \mathrm{~h}$ (or until cells in control populations reached confluence) using the Cytation 5 Cell Imaging Multi-Mode Reader (Biotek) driven 
by Gen5 software (Biotek). A $\times 4$ Plan Fluorite 0.13 NA objective (Olympus) was used to capture images. Between imaging reads, cells were incubated at $37^{\circ} \mathrm{C}$ with $5 \% \mathrm{CO}_{2}$ using the Biospa 8 Automated Incubator (Biotek). Gen5 software (Biotek) was used to process images and to measure cell confluence and the number of cells/ $\mathrm{mm}^{2}$ using high-contrast bright-field images. Parameters including cell size and light-intensity thresholds were specified for each cell line. To determine rates of cell proliferation, the fold change in cells $/ \mathrm{mm}^{2}$ between the first and last reads of each well were calculated and normalized to the control for each experiment. One-way ANOVA with post hoc Tukey's test was used to compare proliferation fold-change values across cell lines to determine statistical significance using Prism 7. For cytotoxicity assays, CellTox ${ }^{\text {tw }}$ Green Dye (Promega, Cat no. G8742) was added to cell media prior to imaging, and the number of cells $/ \mathrm{mm}^{2}$ was recorded for both GFP and bright-field channels. After 4 days of imaging, the area under the proliferation curve for the CellTox-stained cells was divided by the area under the proliferation curve for the total number of cells, and this value was normalized to the control for each cell line as the metric for relative cell death. An unpaired $t$ test was used to determine the significance between control and KIF18A KD for each cell line by comparing the normalized proliferation fold-change values.

\begin{abstract}
Automated cell count validation. Cells were seeded in a series of increasing densities in either a 96- or 24-well dish and allowed to adhere for $24 \mathrm{~h}$. Cells were then incubated with Hoechst stain (Invitrogen, Cat no. H3570), a cell-permeable nuclear dye, for 30 min before being imaged using the Cytation 5 system, as described previously. For each field, one high-contrast bright-field image and one fluorescence image were acquired, and Gen5 software was used to process images and analyze the number of cells $/ \mathrm{mm}^{2}$ using the parameters defined in the proliferation assays. The correlation between cell densities measured in the bright-field images and the fluorescence images was graphed as a scatterplot (Supplementary Fig. 2).
\end{abstract}

Immunofluorescence. Cells were grown on glass coverslips and fixed using either $-20^{\circ} \mathrm{C}$ methanol or $1 \%$ paraformaldehyde in $-20^{\circ} \mathrm{C}$ methanol. Cells were blocked with $20 \%$ goat serum in antibody diluting buffer (Abdil- TBS, $1 \%$ BSA, $0.1 \%$ Triton $\mathrm{X}-100$, and $0.1 \%$ sodium azide) and incubated with the following primary antibodies: mouse anti- $\alpha$-tubulin (DM1 $\alpha$ ) 1:500 (Millipore Sigma, Cat no. T6199) for one hour at room temperature (RT), human anti-centromere antibody (ACA) 1:250 (antibodies Incorporated, Cat no. 15-235) overnight at $4{ }^{\circ} \mathrm{C}$, rabbit anti- $\gamma$ tubulin 1:500 (Abcam, Cat no. ab11317) for $1 \mathrm{~h}$ at RT, mouse anti- $\gamma$-tubulin 1:500 (Sigma-Aldrich, Cat no. T5326) for $1 \mathrm{~h}$ at RT, rabbit anti-KIF18A 1:100 (Bethyl Laboratories, Cat no. A301-080A) at $4{ }^{\circ} \mathrm{C}$ overnight, mouse anti-centrin-1 1:500 (Millipore Sigma, Cat no. 04-1624) for one hour at RT, rabbit anti-mCherry 1:500 (Abcam, Cat no. ab167453) for $1 \mathrm{~h}$ at RT, and rabbit anti-KIF18B $1: 2000^{52}$ for $1 \mathrm{~h}$ at RT. Secondary antibodies conjugated to Alexa Fluor 488, 594, and 647 (Molecular Probes) were used at concentrations of 1:15,000 for $1 \mathrm{~h}$ at RT. Coverslips were mounted onto glass slides using Prolong Gold anti-fade mounting medium with DAPI (Molecular Probes).

Microscopy. Fixed and live-cell images were acquired using a Ti-E or Ti-2E inverted microscope (Nikon Instruments) driven by NIS Elements software (Nikon Instruments). Images were captured using a Clara cooled charge-coupled device (CCD) camera (Andor) or Prime Bsi sCMOS camera (Teledyne Photometrics) with a Spectra-X light engine (Lumencore). For live-cell imaging, cells in $\mathrm{CO}_{2}$-independent media (Gibco) were imaged using Nikon objectives Plan Apo $20 \times 0.75$ NA or $40 \times 0.95 \mathrm{NA}$ and an environmental chamber at $37^{\circ} \mathrm{C}$. Fixed-cell images were taken using Plan Apo $40 \times 0.95$ NA, Plan Apo $\lambda 60 \times 1.42$ NA, and APO $100 \times 1.49$ NA (Nikon).

Western blot. Cells were lysed in PHEM lysis buffer (60 mM Pipes, $10 \mathrm{mM}$ EGTA, $4 \mathrm{mM} \mathrm{MgCl}_{2}$, and $25 \mathrm{mM} \mathrm{HEPES}$ ) with $1 \%$ Triton X-100 and protease inhibitors, incubated on ice for $10 \mathrm{~min}$, and centrifuged at maximum speed for $5 \mathrm{~min}$. Laemmli buffer with $\beta$-mercaptoethanol was added to the supernatant prior to boiling for $10 \mathrm{~min}$ at $95^{\circ} \mathrm{C}$. Lysates were run on $4-15 \%$ gradient gels (BioRad), transferred (75 min at $100 \mathrm{~V}$ ) to PVDF membrane (BioRad), and blocked for $1 \mathrm{~h}$ in 1:1 Odyssey Blocking Buffer (Li-Cor) and TBS with $0.1 \%$ Tween-20. Membranes were incubated with primary antibodies overnight at $4{ }^{\circ} \mathrm{C}$. Primary antibodies included 1:1000 mouse anti-GAPDH (Millipore, Cat no. MAB374), 1:500 rabbit anti-KIF18A (Bethyl Laboratories, Cat no. A301-080A), 1:1000 rabbit anti-Kif4A (Bethyl Laboratories, Cat no. A301-074A), 1:1000 rabbit anti-KIF22 (Millipore Sigma, Cat no. HPA048213), 1:1000 rabbit anti-MCAK (Abcam, Cat no. ab187652), 1:1000 rabbit anti-MAD2 (Bethyl Laboratories, Cat no. A300-301A), and 1:1000 rabbit anti-Cleaved Caspase-3 (Cell Signaling Technology, Cat no. 9661). Secondary antibodies included goat anti-Rabbit IgG DyLight 800 conjugate (Invitrogen, Cat no. SA5-10036) and goat anti-mouse IgG DyLight 680 (Invitrogen, Cat no. SA5-10170), which were each diluted to $1: 15,000$ in 1:1 Odyssey blocking buffer/TBS and added to the membrane for one hour at room temperature. Membranes were imaged using an Odyssey CLx (Li-Cor). Full scans of all blots are available in Source Data.
Live imaging. Cells were plated in a glass-bottom 24 -well dish and treated with the indicated siRNA $\sim 24 \mathrm{~h}$ before imaging. Six hours before imaging, the cell culture media was replaced with $\mathrm{CO}_{2}$-independent media containing $100 \mu \mathrm{M}$ SiR-tubulin (Cytoskeleton, Cat no. CY-SC002). For conditions involving UMK57 (a kind gift from Duane Compton) or DMSO, the specified drug was added to the $\mathrm{CO}_{2-}$ independent media with siR-tubulin. To image centrosomes, MDA-MB-231 cells were transfected with RFP-pericentrin ${ }^{53}$ via a $4 \mathrm{D}$ nucleofector system (Lonza) prio to seeding in glass-bottom dishes, and siRNAs were added $24 \mathrm{~h}$ later. Cells were imaged every $2 \mathrm{~min}$ for $16-20 \mathrm{~h}$ using a $40 \times 0.75 \mathrm{NA}$ objective (Nikon).

KIF2C/MCAK overexpression. MDA-MB-231 cells were transfected with either a construct containing mCherry fused to the N-terminus of full-length KIF2C/MCAK (mCh-MCAK-FL) or a construct containing the centromere binding domain of CENP-B fused to the C-terminus of mCherry and the N-terminus of MCAK (mChCPB-MCAK), both kind gifts from Linda Wordeman ${ }^{41}$. mCh-CPB-MCAK is similar to a previously described construct designed to increase MCAK activity at centromeres (RCPBM) ${ }^{41}$ but contains mCherry instead of mRFP and an additional eight amino acids linking CPB and MCAK to improve expression. Transfections were performed using a $4 \mathrm{D}$ nucleofector system (Lonza) and plated onto 12 -mm glass coverslips. KIF18A or control siRNA was added to cells $24 \mathrm{~h}$ post transfection, and cells were fixed and stained for immunofluorescence after an additional $24 \mathrm{~h}$.

Mitotic timing and mitotic index analyses. To measure the length of mitosis, live cells were imaged every $2 \mathrm{~min}$ for $16-20 \mathrm{~h}$ using differential interference contrast (DIC) microscopy. The time between nuclear envelope breakdown (NEB) and anaphase onset (AO) was used to indicate the time a cell spent in mitosis. Mitotic index was measured using fixed-cell images by counting the number of mitotic cells divided by the total number of cells. All mitotic index fields were taken with a $\times 40$ objective. An unpaired $t$ test was used to determine statistical significance between control and KIF18A KD conditions for each cell line using the mean percentages of mitotic cells from each experimental replicate. Contingency tables were created in Prism 7 to compare the total number of cells that either divided or failed to divide; statistical significance was then determined using Chi-square tests to compare between the conditions within each cell line. An unpaired $t$ test was used to determine the statistical significance between control and KIF18A KD conditions in the mitotic timing analysis by comparing the averages of all the timing values (minutes from NEB to AO) between the two conditions within each cell line. For HeLa Kyoto mitotic timing rescue experiments, a one-way ANOVA was used to compare averages of the timing values between the three conditions in Prism 7.

Mitotic spindle morphology analyses. To analyze mitotic spindle morphology, cells were fixed and stained for $\gamma$-tubulin, $\alpha$-tubulin, and centrin-1. Enough optical slices spaced $200 \mathrm{~nm}$ apart were captured to visualize the entire 3-D structure of the spindle. Spindles with three or more visible $\gamma$-tubulin-containing microtubuleorganizing centers were classified as multipolar. Contingency tables were created in Prism 7 to compare the total number of bipolar and multipolar cells between conditions, and statistical significance was determined using a Chi-square test. To characterize the structure of microtubule-organizing centers across different conditions, pericentriolar material (PCM) and centrioles were stained and imaged in fixed cells. Cells were considered to possess fragmented pericentriolar material if they had supernumerary poles observed via $\gamma$-tubulin staining but lacked centrioles (centrin-1 puncta) at one or more of the poles. An unpaired $t$ test was used to determine statistical significance between control and KIF18A KD conditions for PCM fragmentation measurements. Intercentriolar distance, or the distance in microns between two centrioles in a pair, was measured from the center of one centriole to the center of the adjacent centriole. One-way ANOVA with post hoc Tukey's test was used to compare intercentriolar distance measurements between conditions in Prism 7.

Analysis of live spindle pole dynamics. Movies of MDA-MB-231 cells stained with SiR-tubulin were analyzed to assess the timing of multipolar spindle formation. If a cell entered mitosis with three or more visible microtubule-organizing centers, it was considered to have begun multipolar. If a cell entered mitosis with two microtubule-organizing centers but ended up with three or more by the end of the movie, it was considered to have split poles. Contingency tables were created in Prism 7 to compare the total number of cells that split poles and the total number that remained bipolar between conditions, and statistical significance was determined using a Chi-square test. The same analysis was conducted to compare differences across conditions in the proportion of cells that began multipolar.

Knockdown quantification analysis. The efficiency of siRNA-mediated kinesin knockdowns was measured via either quantitative western blot or immunofluorescence. ImageJ was used for all quantification. KIF18A knockdown efficiency in CRC cell lines was measured by comparing background-subtracted KIF18A fluorescence intensity in cells treated with control or KIF18A siRNA. In TNBC cell lines, KIF18B knockdown efficiency was measured by comparing backgroundsubtracted KIF18B fluorescence intensity in cells treated with control or KIF18B siRNA. All other knockdown quantifications were determined by western blot analysis. For MCF10A and MDA-MB-231 cell lines, the KIF18A knockdown efficiency was further analyzed at the RNA level by qRT-PCR. 
qRT-PCR. Total RNA extraction was carried out using RNeasy Mini Kit (Qiagen). Extracted RNA was screened by the Vermont Integrative Genomics Resource (VIGR) DNA Facility for purity and integrity using a 2100 Bioanalyzer (Agilent Technologies). Human GAPDH (Hs01015428_m1) and human KIF18A (Hs02786624_g1) Taqman probes and primers (Thermo Fisher Scientific) were used for reverse transcription and qRT-PCR. KIF18A RNA expression levels were normalized to GAPDH RNA levels in each cell line.

Statistics and reproducibility. Data are represented as mean \pm standard deviation (SD) unless otherwise specified. Statistical tests used to analyze each dataset are described in the figure legends. All statistical tests were performed using GraphPad Prism 7.0. Exact values for the number of cells/fields analyzed are provided in the figure legends along with the number of independent experimental replicates analyzed.

\section{Data availability}

The data associated with the study are available within the Article, Supplementary Information, or from the corresponding author upon reasonable request. Source data are provided with this paper.

Received: 11 December 2020; Accepted: 25 January 2021; Published online: 22 February 2021

\section{References}

1. Lengauer, C., Kinzler, K. W. \& Vogelstein, B. Genetic instabilities in human cancers. Nature 396, 643-649 (1998).

2. Bakhoum, S. F., Genovese, G. \& Compton, D. A. Deviant kinetochore microtubule dynamics underlie chromosomal instability. Curr. Biol. 19, 1937-1942 (2009).

3. Ertych, N. et al. Increased microtubule assembly rates influence chromosomal instability in colorectal cancer cells. Nat. Cell Biol. 16, 779-791 (2014).

4. Cimini, D. et al. Merotelic kinetochore orientation is a major mechanism of aneuploidy in mitotic mammalian tissue cells. J. Cell Biol. 153, 517-527 (2001).

5. Bakhoum, S. F., Thompson, S. L., Manning, A. L. \& Compton, D. A. Genome stability is ensured by temporal control of kinetochore-microtubule dynamics. Nat. Cell Biol. 11, 27-35 (2009).

6. Ganem, N. J., Godinho, S. A. \& Pellman, D. A mechanism linking extra centrosomes to chromosomal instability. Nature 460, 278-282 (2009).

7. Bakhoum, S. F. et al. The mitotic origin of chromosomal instability. Curr. Biol. 24, R148-R149 (2014).

8. Lee, A. J. X. et al. Chromosomal instability confers intrinsic multidrug resistance. Cancer Res. 71, 1858-1870 (2011).

9. Bakhoum, S. F. et al. Chromosomal instability drives metastasis through a cytosolic DNA response. Nature 553, 467-472 (2018).

10. Jordan, M. A. \& Wilson, L. Microtubules as a target for anticancer drugs. Nat. Rev. Cancer 4, 253-265 (2004).

11. Weaver, B. A. A. How Taxol/paclitaxel kills cancer cells. Mol. Biol. Cell 25 2677-2681 (2014).

12. Tischer, J. \& Gergely, F. Anti-mitotic therapies in cancer. J. Cell Biol. 218, 10-11 (2019)

13. Zasadil, L. M. et al. Cytotoxicity of paclitaxel in breast cancer is due to chromosome missegregation on multipolar spindles. Sci. Transl. Med. 6, 229ra43-229ra43 (2014).

14. Mitchison, T. J., Pineda, J., Shi, J. \& Florian, S. Is inflammatory micronucleation the key to a successful anti-mitotic cancer drug? R. Soc. Open Biol. 7, 170182 (2017).

15. Lehmann, B. D. et al. Identification of human triple-negative breast cancer subtypes and preclinical models for selection of targeted therapies. J. Clin. Investig. 121, 2750-2767 (2011).

16. Mouradov, D. et al. Colorectal cancer cell lines are representative models of the main molecular subtypes of primary cancer. Cancer Res. 74, 3238-3247 (2014).

17. Mayr, M. I. et al. The human kinesin Kif18A is a motile microtubule depolymerase essential for chromosome congression. Curr. Biol. 17, 488-498 (2007).

18. Stumpff, J., Dassow, G., von, Wagenbach, M., Asbury, C. L. \& Wordeman, L. The kinesin- 8 motor Kif18A suppresses kinetochore movements to control mitotic chromosome alignment. Dev. Cell 14, 252-262 (2008).

19. Czechanski, A. et al. Kif18a is specifically required for mitotic progression during germ line development. Dev. Biol. 402, 253-262 (2015).

20. Fonseca, C. L. et al. Mitotic chromosome alignment ensures mitotic fidelity by promoting interchromosomal compaction during anaphase. J. Cell Biol. 218, 1086-1088 (2019).
21. Janssen, L. M. E. et al. Loss of Kif18A results in spindle assembly checkpoint activation at microtubule-attached kinetochores. Curr. Biol. 28, 2685-2696 (2018)

22. Edzuka, T. \& Goshima, G. Drosophila kinesin-8 stabilizes the kinetochore-microtubule interaction. J. Cell Biol. 218, 474-488 (2018).

23. Zhu, C. et al. Functional analysis of human microtubule-based motor proteins, the kinesins and dyneins, in mitosis/cytokinesis using RNA interference. Mol. Biol. Cell 16, 3187-3199 (2005).

24. Häfner, J., Mayr, M. I., Möckel, M. M. \& Mayer, T. U. Pre-anaphase chromosome oscillations are regulated by the antagonistic activities of Cdk1 and PP1 on Kif18A. Nat. Commun. 5, 4397 (2014).

25. Malaby, H. L., Lessard, D. V., Berger, C. L. \& Stumpff, J. KIF18A's neck linker permits navigation of microtubule-bound obstacles within the mitotic spindle. Life Sci. Alliance 2, e201800169 (2019).

26. Logarinho, E. et al. CLASPs prevent irreversible multipolarity by ensuring spindle-pole resistance to traction forces during chromosome alignment. Nat. Cell Biol. 14, 295-303 (2012).

27. Kwon, M. et al. Mechanisms to suppress multipolar divisions in cancer cells with extra centrosomes. Gene Dev. 22, 2189-2203 (2008).

28. Kleylein-Sohn, J. et al. Acentrosomal spindle organization renders cancer cells dependent on the kinesin HSET. J. Cell Sci. 125, 5391-5402 (2012).

29. Pannu, V. et al. HSET overexpression fuels tumor progression via centrosome clustering-independent mechanisms in breast cancer patients. Oncotarget 6, 6076-6091 (2015)

30. Zhao, X. et al. Host microtubule plus-end binding protein CLASP1 influences sequential steps in the Trypanosoma cruzi infection process. Cell Microbiol. 15, 571-584 (2013).

31. Maiato, H. \& Logarinho, E. Mitotic spindle multipolarity without centrosome amplification. Nat. Cell Biol. 16, 386-394 (2014).

32. Gorbsky, G. J., Chen, R.-H. \& Murray, A. W. Microinjection of antibody to $\mathrm{Mad} 2$ protein into mammalian cells in mitosis induces premature anaphase. J. Cell Biol. 141, 1193-1205 (1998).

33. Stumpff, J., Wagenbach, M., Franck, A., Asbury, C. L. \& Wordeman, L. Kif18A and chromokinesins confine centromere movements via microtubule growth suppression and spatial control of kinetochore tension. Dev. Cell 22, 1017-1029 (2012)

34. Yvon, A.-M. C., Wadsworth, P. \& Jordan, M. A. Taxol suppresses dynamics of individual microtubules in living human tumor cells. Mol. Biol. Cell 10, 947-959 (1999).

35. Jordan, M. A., Thrower, D. \& Wilson, L. Effects of vinblastine, podophyllotoxin and nocodazole on mitotic spindles. Implications for the role of microtubule dynamics in mitosis. J. Cell Sci. 102, 401-416 (1992).

36. Brabander, M. D., Geuens, G., Nuydens, R., Willebrords, R. \& Mey, J. D. Taxol induces the assembly of free microtubules in living cells and blocks the organizing capacity of the centrosomes and kinetochores. Proc. Natl Acad. Sci. USA 78, 5608-5612 (1981).

37. Hornick, J. E. et al. Live-cell analysis of mitotic spindle formation in taxoltreated cells. Cell Motil. Cytoskel. 65, 595-613 (2008).

38. Kapoor, T. M., Mayer, T. U., Coughlin, M. L. \& Mitchison, T. J. Probing spindle assembly mechanisms with monastrol, a small molecule inhibitor of the mitotic kinesin, Eg5. J. Cell Biol. 150, 975-988 (2000).

39. Wordeman, L., Decarreau, J., Vicente, J. J. \& Wagenbach, M. Divergent microtubule assembly rates after short- versus long-term loss of endmodulating kinesins. Mol. Biol. Cell 27, 1300-1309 (2016).

40. Orr, B., Talje, L., Liu, Z., Kwok, B. H. \& Compton, D. A. Adaptive resistance to an inhibitor of chromosomal instability in human cancer cells. Cell Rep. 17, 1755-1763 (2016)

41. Wordeman, L., Wagenbach, M. \& Dassow, G. von. MCAK facilitates chromosome movement by promoting kinetochore microtubule turnover. $J$. Cell Biol. 179, 869-879 (2007).

42. Liu, X. S. et al. Germinal cell aplasia in kif18a mutant male mice due to impaired chromosome congression and dysregulated BubR1 and CENP-E. Genes Cancer 1, 26-39 (2010)

43. Zhu, H. et al. Targeted deletion of Kif18a protects from colitis-associated colorectal (CAC) tumors in mice through impairing Akt phosphorylation. Biochem Bioph Res. Co. 438, 97-102 (2013).

44. Zhang, C. et al. Kif18A is involved in human breast carcinogenesis. Carcinogenesis 31, 1676-1684 (2010).

45. Du, Y., English, C. A. \& Ohi, R. The kinesin-8 Kif18A dampens microtubule plus-end dynamics. Curr. Biol. 20, 374-380 (2010).

46. Stumpff, J. et al. A tethering mechanism controls the processivity and kinetochore-microtubule plus-end enrichment of the kinesin-8 Kif18A. Mol. Cell 43, 764-775 (2011).

47. Quinton, R. J. et al. Whole-genome doubling confers unique genetic vulnerabilities on tumour cells. Nature https://doi.org/10.1038/s41586-020 03133-3 (2021).

48. Cohen-Sharir, Y. et al. Aneuploidy renders cancer cells vulnerable to mitotic checkpoint inhibition. Nature https://doi.org/10.1038/s41586-020-03114-6 (2021). 
49. Khandelia, P., Yap, K. \& Makeyev, E. V. Streamlined platform for short hairpin RNA interference and transgenesis in cultured mammalian cells. Proc. Natl Acad. Sci. USA 108, 12799-12804 (2011).

50. Sturgill, E. G., Norris, S. R., Guo, Y. \& Ohi, R. Kinesin-5 inhibitor resistance is driven by kinesin-12. J. Cell Biol. 213, 213-227 (2016).

51. Kim, H., Fonseca, C. \& Stumpff, J. A unique kinesin- 8 surface loop provides specificity for chromosome alignment. Mol. Biol. Cell 25, 3319-3329 (2014).

52. Shin, Y. et al. Biased Brownian motion as a mechanism to facilitate nanometer-scale exploration of the microtubule plus end by a kinesin-8. Proc. Natl Acad. Sci. USA 112, E3826-E3835 (2015).

53. Gillingham, A. K. \& Munro, S. The PACT domain, a conserved centrosomal targeting motif in the coiled-coil proteins AKAP450 and pericentrin. EMBO Rep. 1, 524-529 (2000).

\section{Acknowledgements}

The authors wish to thank Dr. Carol Vallett, Dr. Marion Thurnauer, Dr. Marie Wood, and Dr. Christopher Anker for insightful discussions and suggestions. We also thank Dr. Uri Ben-David and Dr. Neil Ganem for sharing unpublished results and Dr. Linda Wordeman and Dr. Duane Compton for reagents. This work was supported by Susan G Komen grant CCR16377648, NIH grant GM121491, American Cancer Society Institutional Research Grant 14-196-01, and a Lake Champlain Cancer Research Organization pilot grant (to J.S.) and by Department of Defense PRCRP Horizon Award W81XWH17-1-0371 (to H.M.). The authors declare no competing financial interests.

\section{Author contributions}

C.M., C.L.F., and J.S. contributed to the conceptual design of the project. C.M., C.L.F., K. A.Q., L.W., S.E.V., H.L.H.M., J.E.C., and J.S. designed, performed, and analyzed experiments. K.A.Q., S.E.V., and J.E.C. generated essential reagents and assays. C.M., C.L. F., and J.S. wrote the paper and created all figures. C.M., C.L.F., K.A.Q., L.W., S.E.V., H.L. H.M., J.E.C., and J.S. edited the paper.

\section{Competing interests}

The authors declare no competing interests.

\section{Additional information}

Supplementary information The online version contains supplementary material available at https://doi.org/10.1038/s41467-021-21447-2.

Correspondence and requests for materials should be addressed to J.S.

Peer review information Nature Communications thanks the anonymous reviewer(s) for their contribution to the peer review of this work.

Reprints and permission information is available at http://www.nature.com/reprints

Publisher's note Springer Nature remains neutral with regard to jurisdictional claims in published maps and institutional affiliations.

(c) (i) Open Access This article is licensed under a Creative Commons Attribution 4.0 International License, which permits use, sharing, adaptation, distribution and reproduction in any medium or format, as long as you give appropriate credit to the original author(s) and the source, provide a link to the Creative Commons license, and indicate if changes were made. The images or other third party material in this article are included in the article's Creative Commons license, unless indicated otherwise in a credit line to the material. If material is not included in the article's Creative Commons license and your intended use is not permitted by statutory regulation or exceeds the permitted use, you will need to obtain permission directly from the copyright holder. To view a copy of this license, visit http://creativecommons.org/ licenses/by/4.0/.

(C) The Author(s) 2021 